\title{
Exact results for corner filling on a quadratic lattice
}

\author{
D. B. Abraham \\ Laboratory of Computational Engineering, Helsinki University of Technology, P. O. Box 9203, Helsinki, FIN-02015, Finland and \\ Theoretical Physics, University of Oxford, Oxford OX1 3NP, United Kingdom \\ A. Maciołek \\ Institute of Physical Chemistry, Polish Academy of Sciences, Department III, Kasprzaka 44/52, PL-01-224 Warsaw, Poland
}

(Received 4 April 2005; published 2 September 2005)

\begin{abstract}
An exact statistical mechanical derivation of corner filling in the two-dimensional Ising ferromagnet is given. The surface fields on the edges that meet at the corner are not required to be the same in the solution. Both thermodynamic results and microscopic structure are obtained.
\end{abstract}

DOI: 10.1103/PhysRevE.72.031601

PACS number(s): 68.08.Bc, 05.50.+q, 68.35.Rh

\section{INTRODUCTION}

The significance of the wetting transition in the equilibrium statistical mechanical treatment of surfaces in condensed systems has become increasingly clear over the past 25 years or so [1]. In this case, the wall with which the fluid components interact differentially is treated as otherwise featureless; it is flat. The adsorption properties of chemically and physically modified surfaces are striking and of great theoretical and technical significance [2]. For instance, a striated surface with V-shaped grooves should be completely wetted for a larger region of parameter space, depending on the angle at the apex of the groove, than would be the case for a flat featureless surface [3-6]. There is also an interesting zoological example: the Stenocara beetle uses its back, which is patterned with hydrophilic areas embedded in a hydrophobic one, to concentrate fog in the Namib desert into water droplets which flow under gravity and are then swallowed [7].

The purpose of this paper is to give in some detail the exact solution of a microscopic model which shows both a filling and a wetting transition along with the usual criticality of a lattice gas system equivalent to the uniaxial classical ferromagnet. This is a modification of the planar Ising model which allows Dobrushin boundary conditions to introduce domain walls, or interfaces between pure phases, in a geometrically controlled way, while still maintaining exact solvability. Our starting point is to review what is known about interfacial structure and wetting for the planar Ising model.

We consider a planar rectangular lattice with coordinates $i$ (horizontal) and $j$ (vertical). At each site there is attached a spin $\sigma_{i, j}= \pm$. We denote by $K_{1}$ and $K_{2}$ the coupling constants of interactions along vertical and horizontal bonds, respectively. The configurational Hamiltonian is defined as

$$
H=-K_{1} \sum_{i, j} \sigma_{i, j} \sigma_{i, j+1}-K_{2} \sum_{i, j} \sigma_{i, j} \sigma_{i+1, j}
$$

where the sums run over $1 \leqslant i \leqslant M$ and $1 \leqslant j \leqslant N$. Suppose the lattice is wrapped onto a cylinder, with axis in the $(0,1)$ direction, so that we have cyclic boundary conditions in the $(1,0)$ direction. A key idea, due to Fisher [8], to develop the calculation of surface tension is to fix the spins on the top and bottom of the cylinder, as shown in Fig. 1. The surface tension $\tau(1,0)$ in units of $k_{B} T$ for an interface oriented on average parallel to $(1,0)$ is given by

$$
\tau(1,0)=-\lim \frac{1}{N} \ln \frac{Z^{+-}}{Z^{++}} .
$$

This surface tension was already known to Onsager [9] from a different definition using a lattice with antiferomagnetism and an odd number of spins in one direction to induce an interface in the perpendicular direction. The ultimate significance and interest of Eq. (1.2) is in the precise limiting procedure followed, and further, in the asymptotics leading to it. With the appropriate technical developments, this leads to a rather complete theory of finite size effects, including those resulting from the proliferation of interfaces if the lattice is too acicular [10]. For the present purposes, the limit in Eq. (1.2) can be taken as $N \rightarrow \infty$ followed by $M \rightarrow \infty$. This suffices to suppress configurations with more than one domain wall. The end wall terms and bulk ones in $Z^{+-} / Z^{++}$ should then cancel by symmetry, leaving just the incremental free energy from the interface on taking the logarithm. The result is

$$
\tau(1,0)=\hat{\gamma}(0)
$$

where the Onsager function $\gamma(k)$ is defined by the nonnegative real solution of

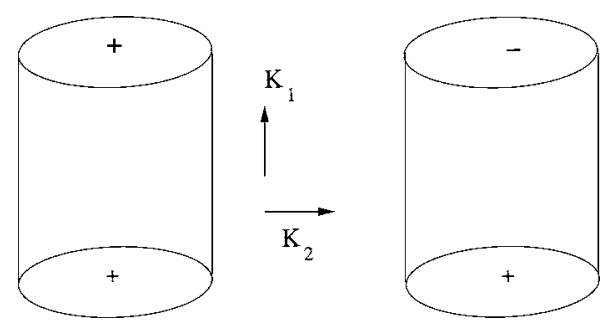

FIG. 1. Two fixed spin boundary conditions on the ferromagnetic cylinder. In the subcritical region, the left hand one has an even number of domain walls, whereas the right hand one has an odd number of tiers, including the fixed spin ones, each of circumference $N$. 


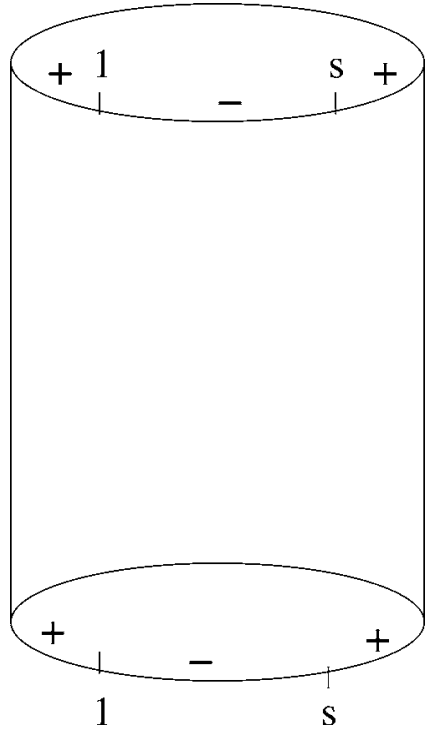

FIG. 2. Dobrushin boundary conditions to introduce domain walls running parallel to the $(0,1)$ axis of a cylinder with fixed ends.

$$
\cosh \gamma(k)=\cosh 2 K_{1}^{*} \cosh 2 K_{2}-\sinh 2 K_{1}^{*} \sinh 2 K_{2} \cos k
$$

for real $k$. The function $\hat{\gamma}$ has the analogous definition but with $K_{1}$ and $K_{2}$ interchanged. The dual variable $K_{j}^{*}$ is given for $K_{j}>0$ by real positive solutions of

$$
e^{-2 K_{j}^{*}}=\tanh K_{j}
$$

Suppose the cylindrical lattice is now modified by introducing a reversal of sign between 1 and $s$ on each edge of the cylinder, as in Fig. 2. Taking $N \rightarrow \infty$ and then $s \rightarrow \infty$ implies that there are two independent domain walls running parallel to the $(0,1)$ axis, with fixed ends. These are the Dobrushin boundary conditions [11]. Notice that $M$ is still finite at this stage. The magnetization along the line $y=M / 2$, assuming $M$ even, has been calculated for all subcritical temperatures, revealing capillary fluctuations; to find the typical intercept of the interface with $y=M / 2$ we have to look on a scale of $M^{1 / 2}$. The result for the magnetization defined in terms of the spin variables $\sigma(x, y)= \pm 1$ is [12]

$$
\begin{aligned}
& \lim \lim \lim \left\langle\sigma\left(\alpha M^{\delta}, M / 2\right)\right\rangle \\
& M \rightarrow \infty s \rightarrow \infty N \rightarrow \infty
\end{aligned}
$$

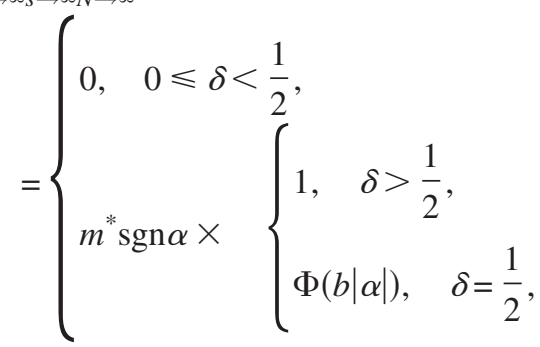

where $m^{*}$ is the spontaneous magnetization, $\Phi(x)$ is the center of the normal distribution, and $b$ is the surface stiffness [13]. Thus, the interface between coexistent phases manifests divergent fluctuations, contrary to the intuitions from thermodynamics and from earlier experimental obser-

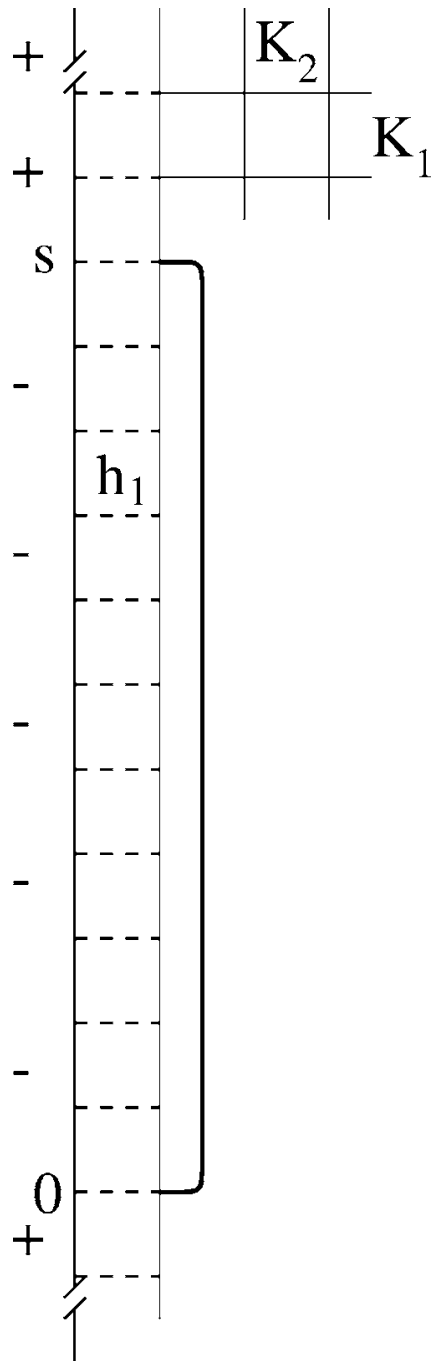

FIG. 3. Semi-infinite Ising lattice (only part of a lattice is shown) with a column of weakened horizontal bonds $h_{1}<K_{2}$ contiguous with the surface, and with boundary conditions that introduce a domain wall pinned to the wall at low temperatures with fixed ends at $(0,0)$ and $(0, s)$.

vations. These have recently been supplemented by direct experimental observation of capillary fluctuations [14]. In ferromagnetic lattice gas models, such fluctuations can be damped by grain boundaries of weakened bonds, bringing in the standard energy-entropy paradigm for phase transitions. Of particular relevance here is the behavior occasioned by terminating both ends of the interface in the edge of a half plane, as shown in Fig. 3. With the introduction of a line of weakened bonds normal to and contiguous with the surface, as $s \rightarrow \infty$, the interface manifests a phase transition at a strictly subcritical temperature given by $w_{1}=1$ with

$$
w_{1}=e^{2 K_{1}}\left(\cosh 2 K_{2}-\cosh 2 h_{1}\right) / \sinh 2 K_{2}
$$

and $0<h_{1}<K_{2}$. The phase diagram is given by solid line in Fig. 4. In the region $w_{1}>1$, the interface is found on average at a finite distance from the wall; it is pinned. Above the transition, the interface depins to a fluctuating regime analo- 


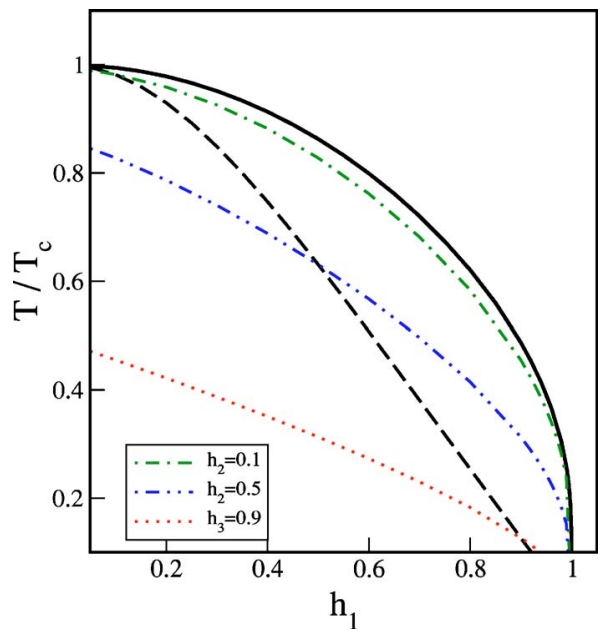

FIG. 4. (Color online) Surface phase diagram for the critical wetting (solid line) in the two-dimensional Ising model. Dashed line is the line of filling transition for the symmetric Ising corner with surface fields $h_{1}=h_{2}=h$. Three cases of nonsymmetric boundary conditions on edges of the corner are also shown. $h_{1}$ is given in units of the reduced coupling constant $K$.

gous to that in (1.6), but with a different $\Phi(x)$ [15]. Thus, the interface notices the wall, even though it is far from it, a subtlety that has been placed on a general footing by Fisher [16]. This phase transition is not the same as wetting, as described in basic chemical physics texts, where the characterization is thermodynamic in terms of the contact angle and Young's relation, defined on any length scale. The existence of capillary fluctuations clearly invalidate such a description. The correct approach is to view the contact angle in an appropriate definition as a spatial average. The Young equation, modified to take into account the angle dependence of surface transition on the lattice, is then recaptured. The contact angle $\Theta_{c}$ satisfies [17]

$$
\left(\cos \Theta_{c}\right) \tau\left(\Theta_{c}\right)-\left(\sin \Theta_{c}\right) \tau^{(1)}\left(\Theta_{c}\right)=f^{x}
$$

where $f^{x}$ is the binding free energy of the interface in the pinning transition given by Eq. (1.7): it takes the simple form

$$
f^{x}=\gamma\left(i v_{1}\right)
$$

where $\exp v_{1}=w_{1}$. The angle-dependent surface tension is given by

$$
\tau(\Theta)=(\cos \theta) \gamma\left[i v_{s}(\Theta)\right]+(\sin \theta) v_{s}(\Theta)
$$

where $v_{s}(\Theta)$ is given by a saddle point calculation as [18]

$$
\gamma^{(1)}\left[i v_{s}(\Theta)\right]=\tan \Theta
$$

The quantity $\gamma^{(1)}(k)$ is evaluated from Eq. (1.4), in a rather implicit form which suffices for Wulff construction calculations [19] of mean Ising "crystallite" shape. It is also of interest to examine Eqs. (1.8) and (1.9) in the context of Eqs. (1.10) and (1.11). This gives

$$
v_{s}\left(\boldsymbol{\Theta}_{c}\right)=v_{1}
$$

where

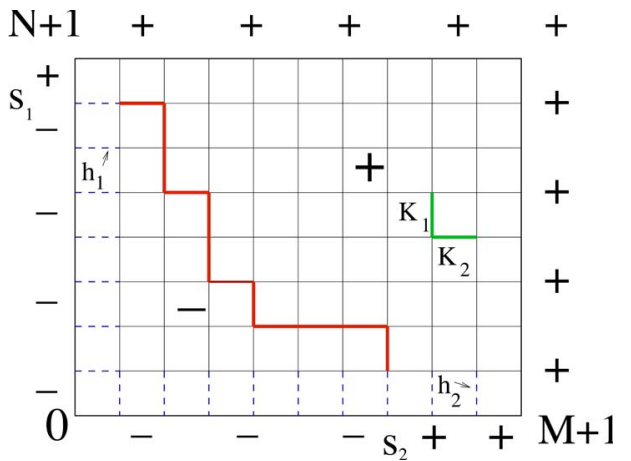

FIG. 5. (Color online) A corner of an Ising lattice with reduced nearest-neighbor couplings $K_{1}$ and $K_{2}$. A row of vertical bonds at the bottom and a column of horizontal bonds at the left edge (dashed lines) are weakened to $h_{1}<K_{1}$ and $h_{2}<K_{2}$ and represent the wall interactions. The boundary conditions impose an interface running from $\left(s_{1}, 1\right)$ to $\left(1, s_{2}\right)$.

$$
\sinh 2 K_{2} \frac{\sinh v_{s}(\Theta)}{(\sinh \gamma)\left[i v_{s}(\Theta)\right]}=\sinh 2 K_{1} \tan \Theta .
$$

Hence $v_{s}(\Theta)$ is an increasing function of $\Theta$; as $v_{1} \searrow 0$ at the pinning-depinning transition, $\Theta_{c} \searrow 0$, as anticipated.

This brings us to the subject of this paper in more detail. What would happen if an interface pinned on $(0,1)$ were joined to one pinned on $(1,0)$ at a corner of an Ising lattice? Referring to Fig. 5, the interface could follow any path from $\left(s_{2}, 0\right)$ to $\left(0, s_{1}\right)$, in particular the direct one at an inclination of $\pi / 4$ to the principal lattice direction. Such a scenario has been considered by Concus and Finn [3] and by Hauge [5], with the suggestion that there is a transition within the pinned regime from interface binding on the walls to the shortest path configuration, on thermodynamic grounds. This would happen if

$$
\sqrt{2} \tau(\pi / 4)=f^{x}(1,0)+f^{x}(0,1)
$$

as has been investigated for $K_{1}=K_{2}$ and $h_{1}=h_{2}$ [20], both using known results for $\tau(\pi / 4)$ and $f^{x}$ and numerically. One of the results is the first exact statistical mechanical investigation of such a phase transition beginning at a microscopic level [21]. At the mesoscopic level, there has been a detailed analysis of solid-on-solid models defined on a wedge, carried out to first order in the departure from linearity [22].

\section{FORMULATION}

Consider a rectangular lattice $(M+1) \times(N+1)$ with spins $\sigma(x, y)= \pm 1$ located at the sites of the lattice interacting with nearest neighbors via the coupling $K_{1}=\beta J_{1}>0$ in the $(0,1)$ direction, and $K_{2}=\beta J_{2}$ in the other. We will consider two different boundary conditions. In the case (a) the surface field $h_{1}>0$ is applied at the left $(x=1)$ and the surface field $h_{2}>0$ at the bottom $(y=1)$ edge of a strip, whereas all spins at the right $(x=M+1)$ and at the top $(y=N+1)$ edges are fixed at the value +1 . In the case (b), the field $h_{1}$ is reversed between points $(1,1)$ and $\left(1, s_{1}\right)$, and the field $h_{2}$ is reversed 
between $(1,1)$ and $\left(s_{2}, 1\right)$, allowing introduction of the domain wall running from $\left(s_{1}, 1\right)$ to $\left(1, s_{2}\right)$ (see Fig. 5). In order to study possible interfacial phase transitions we consider an incremental free energy of that domain wall. It can be calculated from a canonical partition function ratio for the boundary conditions (b) and (a). In units of $k_{B} T$

$$
F\left(s_{1}, s_{2}\right)=-\lim _{N, M \rightarrow \infty} \ln \frac{Z_{M, N}^{b}}{Z_{M, N}^{a}} .
$$

Obviously, bulk contributions to the free energy are the same in both systems. The edge contributions coming from walls exposed to the (-) phase are the same as the ones from walls exposed to the $(+)$ phase; hence they also drop out in Eq. (2.1) and we are left with the excess free energy per unit area of the interface running from $\left(s_{1}, 1\right)$ to $\left(1, s_{2}\right)$.

To calculate $F\left(s_{1}, s_{2}\right)$ exactly we use the transfer matrix technique.

\section{A. Transfer matrix}

We consider the transfer matrix working in the $(0,1)$ direction. It is convenient to replace the surface field $h_{1}$ applied on the left $(x=1)$ edge by a column of modified bonds to additional wall spins located at $(x=0)$ with the interaction $\sum_{y} h_{1} \sigma(0, y) \sigma(1, y)$, and the spins on the additional column at $x=0$ and at the column $x=M+1$ are fixed at the same value, i.e., $\sigma(0, y)=\sigma(M+1, y)$ for all $y$ (see Fig. 5). In order to force the latter we assume that the interaction constant in columns 0 and $M+1$ has a value $K_{0}=\beta J_{0}$, which will become infinite before $N, M \rightarrow \infty$. Thus we are left with $M$ "working" spins.

The elements of the matrix $T_{1}\left(\sigma_{i}, \sigma_{i}^{\prime}\right)$ are indexed by the possible configurations $\sigma_{i}, \sigma_{i}^{\prime}$ of two adjacent rows of spins and they are equal to the contribution to the Boltzmann factor coming from interaction between these rows. In the bulk $T_{1}$ is given by

$$
T_{1}\left(\sigma_{i}, \sigma_{i}^{\prime}\right)=\exp \left(K_{0} \sigma_{0} \sigma_{0}^{\prime}+K_{1} \sum_{i=1}^{M} \sigma_{i} \sigma_{i}^{\prime}+K_{0} \sigma_{M+1} \sigma_{M+1}^{\prime}\right) .
$$

The matrix $T_{2}\left(\sigma_{i}, \sigma_{i}^{\prime}\right)$ accounts for the Boltzmann factors coming from interactions within a single row. $T_{2}$ is a diagonal matrix given by

$$
T_{2}\left(\sigma_{i}, \sigma_{i}^{\prime}\right)=\mathbf{1}\left(\sigma_{i}, \sigma_{i}^{\prime}\right) \exp \left(h_{1} \sigma_{0} \sigma_{1}+K_{2} \sum_{i=2}^{M} \sigma_{i} \sigma_{i+1}^{\prime}\right) .
$$

The transfer matrices can be expressed in terms of Pauli spin operators as follows. $T_{1}$ and $T_{2}$ define operators on the $2^{M+2}$-dimensional vector space $X$ which is the tensor product of $(M+2)$ two-dimensional vector spaces. Each of these twodimensional vector spaces corresponds to the particular spin in the row. The spin operators $\sigma_{j}^{\alpha}$, where $\alpha=x, y, z$, acting on $X$ are defined by

$$
\sigma_{j}^{\alpha}=\left(\otimes_{0}^{j-1} \mathbf{1}\right) \otimes \sigma^{\alpha} \otimes\left(\otimes_{j+1}^{M+1} \mathbf{1}\right)
$$

where the $\sigma^{\alpha}$ are Pauli spin operators,

$$
\sigma^{x}=\left(\begin{array}{cc}
1 & 0 \\
0 & -1
\end{array}\right), \quad \sigma^{y}=\left(\begin{array}{cc}
0 & -i \\
i & 0
\end{array}\right), \quad \sigma^{z}=\left(\begin{array}{cc}
0 & -1 \\
-1 & 0
\end{array}\right),
$$

and $\mathbf{1}$ is a unit matrix. The transfer operator $V_{2}$, defined by $T_{2}$, is given by

$$
V_{2}=\exp \left(h_{1} \sigma_{0}^{x} \sigma_{1}^{x}+K_{2} \sum_{j=1}^{M} \sigma_{m}^{x} \sigma_{m+1}^{x}\right)
$$

The transfer operator $V_{1}$, defined by $T_{1}$, is given by

$$
V_{1}=\prod_{j=1}^{M}\left(e^{K_{1}} \mathbf{1}-e^{-K_{1}} \sigma_{j}^{z}\right)
$$

where we took the limit $K_{0} \rightarrow \infty$. Using the relation

$$
e^{K_{1}}-e^{-K_{1}} \sigma^{z}=\left(2 \sinh 2 K_{1}\right)^{1 / 2} e^{-K_{1}^{*} \sigma^{z}}
$$

$V_{1}$ can be written as

$$
V_{1}=\left(2 \sinh 2 K_{1}\right)^{M / 2} \exp \left(-K_{1}^{*} \sum_{j=1}^{M} \sigma_{j}^{z}\right)
$$

Both operators $V_{1}$ and $V_{2}$ are self-adjoint.

In terms of the transfer matrix operators the incremental can be written as

$$
F^{x}\left(s_{1}, s_{2}\right)=-\lim _{N, M \rightarrow \infty} \ln \frac{\left\langle\Phi_{\infty}\left|R\left(0, s_{2}\right) V_{1}\left(h_{2}^{*}\right)\left(V_{2} V_{1}\right)^{s_{1}} R(0,0)\left(V_{2} V_{1}\right)^{N-1-s_{1}}\right| \Phi_{\infty}\right\rangle}{\left\langle\Phi_{\infty}\left|V_{1}\left(h_{2}^{*}\right)\left(V_{2} V_{1}\right)^{N-1}\right| \Phi_{\infty}\right\rangle} .
$$

$\left|\Phi_{\infty}\right\rangle$ denotes the boundary state that is identical at bottom $(y=0)$ and top $(y=N)$ rows with all spins fixed at the same value. The bonds between the bottom and the first rows are modified and equal to $h_{2}$. The transfer matrix
$V_{1}\left(h_{2}^{*}\right)=\exp \left(-h_{2}^{*} \sum_{j=1}^{M} \sigma_{j}^{z}\right)$, where the dual surface field $h_{2}^{*}$ is defined by $\exp \left(-2 h_{2}^{*}\right)=\tanh h_{2}$, accounts for the Boltzmann factor coming from interactions between these rows. The factor $\left(2 \sinh 2 K_{1}\right)^{(M)(N-1) / 2}\left(2 \sinh 2 h_{2}\right)^{M}$ and the interaction 
energy in end rows is canceled out between the nominator and the denominator. The rotation operator $R^{\prime}\left(0, s_{2}\right)$ could be defined as

$$
R^{\prime}\left(0, s_{2}\right)=\prod_{m=0}^{s_{2}}\left(-\sigma_{m}^{z}\right)
$$

so that

$$
\sigma_{j}^{x} R^{\prime}\left(0, s_{2}\right)=R^{\prime}\left(0, s_{2}\right)\left\{\begin{array}{l}
\sigma_{j}^{x}, \quad s_{2}<j \leqslant M, \\
-\sigma_{j}^{x}, \quad 0 \leqslant j \leqslant s_{2} .
\end{array}\right.
$$

This is a product of $2 s_{2}$ spinors. It is much more convenient, indeed it is crucial, to consider $R^{\prime}\left(0, s_{2}\right)$ operating on the state with all spins fixed up $|+\rangle$. Then Eq. (2.10) can be replaced by

$$
R\left(0, s_{2}\right)=\prod_{m=0}^{s_{2}}\left(-\sigma_{m}^{z}\right) \sigma_{s_{2}+1}^{x}
$$

since $\sigma_{j}^{x}|+\rangle=|+\rangle$ for all $j$. This is linear in the spinors that we are about to define by Jordan-Wigner transformation.

For the analysis of Eq. (2.9) it is useful to introduce the symmetrized form $V^{\prime}=V_{1}^{1 / 2} V_{2} V_{1}^{1 / 2}$. Equation (2.9) can be rewritten in terms of these symmetrized transfer matrices. Using

$$
\left(V_{2} V_{1}\right)^{N-1}=V_{1}^{-1 / 2}\left(V^{\prime}\right)^{N-1} V_{1}^{1 / 2}
$$

one obtains

$$
\begin{aligned}
& F^{x}\left(s_{1}, s_{2}\right)= \\
& \quad-\lim _{N, M \rightarrow \infty} \ln \frac{\left\langle\Phi_{\infty}\left|\widetilde{V_{1}} \widetilde{R}\left(0, s_{2}\right)\left(V^{\prime}\right)^{s_{1}} R(0,0)(V)^{\prime N-1-s_{1}} V_{1}^{1 / 2}\right| \Phi_{\infty}\right\rangle}{\left\langle\Phi_{\infty}\left|\widetilde{V}_{1}(V)^{\prime N-1} V_{1}^{1 / 2}\right| \Phi_{\infty}\right\rangle},
\end{aligned}
$$

where $\widetilde{V}_{1} \equiv V_{1}\left(h_{2}^{*}-K_{1}^{*} / 2\right)$ and $\widetilde{R}\left(0, s_{2}\right) \equiv \tilde{V}_{1}^{-1} R\left(0, s_{2}\right) \widetilde{V}_{1}$.

\section{B. Fermions and the spectrum of the transfer matrix}

The spectrum of the transfer matrix with two equal boundary fields $h_{1}$ was obtained by the extension of the method of Kaufman [23] in Ref. [24]. Below we summarize the results in the lattice-fermion language for the case when one boundary field is equal to $h_{1}$ and the other is equal to $K$ (fixed spins).

The mapping to a theory of free fermions is the core of the form-factor approach in calculating thermodynamic properties of the Ising system which will be employed in this paper. First we define a Jordan-Wigner transformation [25] which turns operators $\sigma_{j}^{\alpha}$ into fermions:

$$
f_{j}=\left(-\sigma_{0}^{z}\right)\left(-\sigma_{1}^{z}\right) \cdots\left(-\sigma_{j-1}^{z}\right) \frac{\sigma_{j}^{x}-i \sigma_{j}^{y}}{2}, \quad j=0, \ldots, M+1 .
$$

$f_{j}$ and the adjoint $f_{j}^{\dagger}$ satisfy anticommutation relations $\left[f_{j}^{\dagger}, f_{k}\right]_{+}=\delta_{j k}$ and $\left[f_{j}, f_{k}\right]_{+}=0$. The fermionic creation operators $f_{j}^{\dagger}$ and annihilation operators $f_{j}$ defined above give the
Fock-space representation of the Hilbert space, where states are generated from the $f$ vacuum, denoted by $|0\rangle$ and defined by the condition $f_{j}|0\rangle=0$ for all $j$, by application of creation operators. The $f$ vacuum corresponds to a free column, i.e., a column of independent free spins. We now define spinor operators in terms of which both transfer matrices take convenient forms. The spinor operators $\Gamma_{-1}, \Gamma_{0}, \ldots, \Gamma_{2(M+1)}$ associated with the above fermionic operators are defined by [23]

$$
\begin{gathered}
\Gamma_{2 j}=-i\left(f_{j}^{\dagger}-f_{j}\right)=\prod_{i=0}^{j-1}\left(-\sigma_{i}^{z}\right) \sigma_{j}^{y}, \quad j=1, \ldots, M+1, \\
\Gamma_{2 j-1}=f_{j}^{\dagger}+f_{j}=\prod_{i=0}^{j-1}\left(-\sigma_{i}^{z}\right) \sigma_{j}^{x}, \quad j=1, \ldots, M+1,
\end{gathered}
$$

and $\Gamma_{-1}=\sigma_{0}^{x}, \Gamma_{0}=\sigma_{0}^{y}$. The spinors satisfy the anticommutation relations $\left[\Gamma_{i}, \Gamma_{j}\right]_{+}=2 \delta_{i j} \mathbf{I}$. In terms of them

$$
\sigma_{j}^{z}=-i \Gamma_{2 j-1} \Gamma_{2 j}, \quad j=0, \ldots, M+1,
$$

and

$$
\sigma_{j}^{x} \sigma_{j+1}^{x}=i \Gamma_{2 j} \Gamma_{2 j+1}, \quad j=0, \ldots, M,
$$

so that

$$
\begin{gathered}
V_{1}=\exp \left(i K_{1}^{*} \sum_{j=1}^{M} \Gamma_{2 j-1} \Gamma_{2 j}\right), \\
V_{2}=\exp \left(i h_{1} \Gamma_{0} \Gamma_{1}+i K_{2} \sum_{j=1}^{M} \Gamma_{2 j} \Gamma_{2 j+1}\right) .
\end{gathered}
$$

Note also that Eq. (2.12) becomes

$$
R\left(0, s_{2}\right)=\Gamma_{2 s_{2}+1} .
$$

The spinor method makes use of the fact that the transfer matrices $V_{i}, i=1,2$, due to their quadratic structure in spinors, are spin representations of a simple $2(M+2)$-dimensional rotation, i.e., the adjoint action of $V_{i}, i=1,2$, on $\Gamma^{T}$ is

$$
V_{i} \Gamma^{T} V_{i}^{-1}=\Gamma^{T} R_{i},
$$

where $R_{i}$ are the $2(M+2) \times 2(M+2)$ rotational matrices satisfying the relations $R_{i}=R_{i}^{\dagger}$ and $R_{i} R_{i}^{T}=1$. The eigenvectors of the matrix $R_{1}$ which represents the adjoint action of $V_{1}$ are

$$
V_{1} f_{j}^{\dagger} V_{1}^{-1}=e^{-2 K_{1}^{*}} f_{j}^{\dagger}, \quad V_{1} f_{j} V_{1}^{-1}=e^{2 K_{1}^{*}} f_{j} .
$$

The eigenstates of $V_{1}$ are the Fock states $f_{j_{1}}^{\dagger} f_{j_{2}}^{\dagger} \cdots f_{j_{p}}^{\dagger}|0\rangle$, and the maximum eigenvalue corresponds to the $f$ vacuum $|0\rangle$.

The eigenvectors of $R_{2}$ are obtained from

$$
\begin{gathered}
V_{2} g_{j}^{\dagger} V_{2}^{-1}=e^{-2 K_{2}} g_{j}^{\dagger}, \quad V_{2} g_{j} V_{2}^{-1}=e^{2 K_{2}} g_{j}, \quad j=1, \ldots, M, \\
V_{2} g_{0}^{\dagger} V_{2}^{-1}=e^{-2 h_{1}} g_{0}^{\dagger}, \quad V_{2} g_{0} V_{2}^{-1}=e^{2 h_{1}} g_{0},
\end{gathered}
$$




$$
V_{2} \Gamma_{-1} V_{2}^{-1}=\Gamma_{-1}, \quad V_{2} \Gamma_{2 M+2} V_{2}^{-1}=\Gamma_{2 M+2},
$$

where the new set of fermionic annihilators $g_{j}$ and creators $g_{j}^{\dagger}$ is defined by

$$
\Gamma_{2 j+1}=g_{j}^{\dagger}+g_{j}, \quad \Gamma_{2 j}=i\left(g_{j}^{\dagger}-g_{j}\right) .
$$

Since no $g_{M+1}$ is defined by Eq. (2.28) there should be two $g$ vacua. Linear combinations of these vacua give two states that correspond to a row with all spins fixed at the same value +1 or -1 .

It follows that the adjoint action of the transfer operator $V^{\prime}$ generates a matrix $R \equiv R_{1}^{1 / 2} R_{2} R_{1}^{1 / 2}$, where $R=R^{\dagger}$. Thus $R$ has real positive eigenvalues $\lambda$ which occur in pairs $\lambda>1$ and $\lambda^{-1}$, with eigenvectors

$$
\begin{gathered}
R y=\lambda y, \\
R y^{*}=\lambda^{-1} y^{*} .
\end{gathered}
$$

The matrix $R$ has unit elements at the top left and bottom right positions, with no other nonzero entry in its border. This means we can remove $\Gamma_{-1}$ and $\Gamma_{2 M+2}$ from the spectral calculation for $R$ for the time being and treat a $2(M+1)$ $\times 2(M+1)$ matrix referring to the action on $\Gamma_{0}, \ldots, \Gamma_{2 M+1}$. The determination of the spectrum of the reduced $R$ can be divided into logical steps. First, there is the "bulk" behavior away from the border, or ends of the column matrix $y$. This is just like that for the transfer matrix with cyclic boundary conditions, so it is no surprise that

$$
\left(\begin{array}{c}
y_{2 j-1} \\
y_{2 j}
\end{array}\right)=e^{i j k}\left(\begin{array}{c}
e^{i \delta^{\prime}(k)} \\
-i
\end{array}\right)
$$

with

$$
e^{i \delta^{\prime}(k)}=(A B)^{-1 / 2}\left[\frac{\left(e^{i k}-A\right)\left(e^{i k}-B\right)}{\left(e^{i k}-A^{-1}\right)\left(e^{i k}-B^{-1}\right)}\right]^{1 / 2}
$$

where $A=\exp 2\left(K_{1}+K_{2}^{*}\right)$ and $B=\exp 2\left(K_{1}-K_{2}^{*}\right)$ and

$$
R y(k)=e^{\gamma(k)} y(k) .
$$

$\gamma(k)$ is the Onsager function from Eq. (1.4), with $\gamma(k)>0$ for real $k$ and $\delta^{\prime}(0)=\pi$ for $B>1 ; B>1$ indicates subcriticality.

Since $\gamma(k)=\gamma(-k)$, linear combinations of eigenvectors in Eq. (2.31) are also "bulk" eigenvectors with the same eigenvalue $\exp \gamma(k)$, the choice of which, together with special assignments of $y_{0}$ and $y_{2 M+1}$, allows us to solve the eigenvalue problem. The result is

$$
\begin{gathered}
y_{2 j-1}(k)=e^{i \delta(k)} e^{-i(j-1) k}+e^{i \delta^{\prime}(k)} e^{i j k}, \\
i y_{2 j}(k)=e^{i \delta(k)} e^{i \delta^{\prime}(k)} e^{-i(j-1) k}+e^{i j k}
\end{gathered}
$$

for $j=1, \ldots, M$ with

$$
\begin{gathered}
y_{0}(k)=i \frac{\sinh 2 h_{1} \cosh K_{1}^{*}}{\sinh \gamma(k)} y_{1}(k), \\
y_{2 M+1}(k)=i \frac{\sinh 2 K_{2} \cosh K_{1}^{*}}{\sinh \gamma(k)} y_{2 M}(k) .
\end{gathered}
$$

Finally, the discretization condition for $k$ follows:

$$
e^{2 i M k}=-e^{i \delta(k)} e^{i\left(\delta^{\prime}(k)-k\right)}
$$

The phase shift factor in Eqs. (2.34) and (2.35) is given by

$$
e^{i \delta(k)}=e^{i \delta^{\prime}(k)}\left(w_{1} e^{i k}-1\right) /\left(e^{i k}-w_{1}\right),
$$

where $w_{1}$ is the wetting parameter introduced in Eq. (1.7).

If $k_{j}$ are the distinct solutions of Eq. (2.38), then $V^{\prime}$ has the form

$$
V^{\prime}=\exp \left(-(1 / 2) \sum_{j=0}^{M+1} \gamma(k)\left[2 X(k)^{\dagger} X(k)\right]-I\right),
$$

where

$$
X(k)=N(k) \sum_{j=0}^{2 M+1} y_{j}(k) \Gamma_{j},
$$

where $N(k)$ are normalization factors. $|\Phi\rangle$ is a vacuum determined by $X(k)|\Phi\rangle=0$ for all $k$.

Now we return to solving Eq. (2.38) with Eq. (2.39). This means searching for the $(2 M+4)$ zeros of a polynomial of this degree, constructing by rationalizing Eq. (2.38). Two of these, $z= \pm 1$, are trivial in the eigenvalue problem. This leaves $2(M+1)$ zeros. With $w_{1}>1$, provided $M$ is big enough there is a pair of purely imaginary $k$ solutions $k= \pm i v$. The real $k$ solutions are easily again found, by graphical inspection, this time more directly from

$$
\tan M k=\tan \frac{\delta(k)+\delta^{\prime}(k)+\pi-k}{2} .
$$

Such investigations are readily fortified with a little real analysis. There is a real root in each interval $(j-1) / M \pi$ $<k_{j}<j / M \pi$ for $j=1, \ldots, M$. The final root that completes the set of $(M+1)$ distinct eigenvectors is given by $k_{0}=i v_{0}^{\prime}$ with

$$
v_{0}^{\prime}=v_{1}+2 e^{-2 M v_{1}} e^{-\delta^{\prime}\left(i v^{\prime}\right)} e^{v_{1}} \sinh v_{1}+O\left(e^{-4 M v_{1}}\right)
$$

where $v_{1}$ is given in terms of Eq. (1.7) by $w_{1}=\exp v_{1}$.

\section{Boundary conditions}

The transfer matrix spectrum has been constructed for the case with hard edges causing the spins in a given edge to align. But the relative spin states in opposite edges are still undetermined, which is remedied as follows. Note that neither $\Gamma_{-1}$ nor $\Gamma_{2 M+2}$ occurs in $V^{\prime}$. There are associated Fermi operators

$$
X_{0}=\frac{1}{2}\left(\Gamma_{-1}-i \Gamma_{2 M+2}\right)
$$

and

$$
X_{0}^{\dagger}=\frac{1}{2}\left(\Gamma_{-1}+i \Gamma_{2 M+2}\right)
$$

and vacuum 


$$
|\hat{\Phi}\rangle=X_{0}|\Phi\rangle
$$

with $|\Phi\rangle$ as constructed above. Arguing by continuity from infinite temperature $T \rightarrow \infty$ state, the parity behavior is

$$
P|\hat{\Phi}\rangle=-|\hat{\Phi}\rangle,
$$

where the parity operator is $P=\prod_{m=0}^{M+1}\left(-\sigma_{m}^{z}\right)$. The eigenvectors $|\hat{\Phi}\rangle$ and $X_{0}^{\dagger}|\hat{\Phi}\rangle$ are degenerate and appropriate linear combinations will specify the fixed edge spin states $|++\rangle$ and $|--\rangle$. Notice that

$$
\sigma_{0}^{x}=X_{0}+X_{0}^{\dagger}, \quad \sigma_{M+1}^{x}=\left(X_{0}-X_{0}^{\dagger}\right) P,
$$

so applying the projectors $P_{j}( \pm)=\left(1 \pm \sigma_{j}^{x}\right) / 2$ with $j=0, M+1$ gives

$$
|++\rangle=2^{-1 / 2}\left(1+X_{0}^{\dagger}\right)|\hat{\Phi}\rangle
$$

and

$$
|--\rangle=2^{-1 / 2}\left(1-X_{0}^{\dagger}\right)|\hat{\Phi}\rangle .
$$

With the notation

$$
\left|(k)_{n} ;++\right\rangle=X^{\dagger}\left(k_{1}\right) \cdots X^{\dagger}\left(k_{n}\right)|++\rangle,
$$

the result

$$
\left[\sigma_{0}^{x}, X^{\dagger}(k)\right]_{+}=0
$$

implies that

$$
\sigma_{0}^{x}\left|(k)_{n} ;++\right\rangle=(-1)^{n}\left|(k)_{n} ;++\right\rangle .
$$

Thus the set of states for $(++)$ or (--) boundary conditions has $n$ even, but for $(-+), n$ must be odd; the maximal eigenvectors for $(+-)$ or $(-+)$ boundary conditions are not found in the subspace spanned by $|\hat{\Phi}\rangle$ and $X_{0}^{\dagger}|\hat{\Phi}\rangle$. This state of affairs is a natural topological constraint given the JordanWigner tail in the fermionic definition (2.15).

\section{DETERMINATION OF THE FORM FACTORS}

The spectral decomposition of the transfer matrix reduces the calculation of the incremental free energy (2.14) to determination of the form factors $\left\langle\Phi_{\infty}\left|\widetilde{V}_{1} X^{\dagger}\left(k_{1}\right) X^{\dagger}\left(k_{2}\right)\right| \Phi\right\rangle$ for all real and imaginary wave vectors. In order to calculate form factors we use the basic equation

$$
\left\langle\Phi_{\infty}\right|\left(\Gamma_{2 j}+i \Gamma_{2 j+1}\right)=0, \quad j=1, \ldots, M-1,
$$

which follows because spins are parallel in $\left\langle\Phi_{\infty}\right|$, which implies that $\left\langle\Phi_{\infty}\right|$ is an eigenvector of $\sigma_{j}^{x} \sigma_{j+1}^{x}$ or equivalently of $i \Gamma_{2 j} \Gamma_{2 j+1}$, with eigenvalue 1. Equation (3.1) follows from

$$
\begin{aligned}
\left\langle\Phi_{\infty}\right| \prod_{0}^{s}\left(-\sigma_{j}^{z}\right) \sigma_{s+1}^{x}= & \left\langle\Phi_{\infty}\right| \Gamma_{2 s+1} \\
& =(-i)\left\langle\Phi_{\infty}\right| \Gamma_{2 s} \sigma_{s}^{x} \sigma_{s+1}^{x} \\
& =-i\left\langle\Phi_{\infty}\right| \Gamma_{2 s} .
\end{aligned}
$$

It follows directly that

$$
\left\langle\Phi_{\infty}\right| \widetilde{V}_{1} \mathcal{O}_{j}=0, \quad j=1, \ldots, M-1,
$$

where

$$
\begin{aligned}
\mathcal{O}_{j}= & \widetilde{V}_{1}^{-1}\left(\Gamma_{2 j}+i \Gamma_{2 j+1}\right) \widetilde{V}_{1}=i(\cosh \vartheta)\left\{\Gamma_{2 j+1}-(\tanh \vartheta) \Gamma_{2 j-1}\right. \\
& \left.+i\left[(\tanh \vartheta) \Gamma_{2 j+2}-\Gamma_{2 j}\right]\right\}
\end{aligned}
$$

with $\vartheta=2 h_{2}^{*}-K_{1}^{*}$. For any eigenvector $X^{\dagger}\left(k_{1}\right) \cdots X^{\dagger}\left(k_{n}\right)|\Phi\rangle$ of the transfer matrix $V^{\prime}$, it follows that

$$
\left\langle\Phi_{\infty}\left|\tilde{V}_{1} \mathcal{O}_{j} X^{\dagger}\left(k_{1}\right) \cdots X^{\dagger}\left(k_{n}\right)\right| \Phi\right\rangle=0, \quad j=1, \ldots, M-1 .
$$

We now expand $\mathcal{O}_{j}$ in the $X(k)$ and $X^{\dagger}(k)$, which is certainly possible because of the linear dependence of the $\Gamma_{j}$ on these "diagonalizing" operators; this gives

$$
-\mathcal{O}_{j}=(\cosh \vartheta) \sum_{k} N(k)\left[A_{j}(k) X^{\dagger}(k)+B_{j}(k) X(k)\right]
$$

where the sum includes real wave numbers in $0<k<\pi$ and the purely imaginary wave number, and the expansion coefficients are given by

$$
A_{j}(k)=\chi(k)\left(e^{-i j k} e^{i \delta}-e^{i j k}\right)
$$

and

$$
B_{j}(k)=\chi_{1}(k)\left(e^{i j k} e^{-i \delta}+e^{-i j k}\right)
$$

with $e^{i \delta}$, which occurs in the "quantization" condition (2.38), being defined by Eq. (2.39). Finally, the functions $\chi(k)$ and $\chi_{1}(k)$, which contain $h_{2}$, are given by

$$
\chi(k)=1-e^{i \delta^{\prime}} e^{i k}+(\tanh \vartheta)\left(e^{i \delta^{\prime}}-e^{i k}\right)
$$

and

$$
\chi_{1}(k)=1+e^{-i \delta^{\prime}} e^{-i k}-(\tanh \vartheta)\left(e^{-i \delta^{\prime}}-e^{-i k}\right) .
$$

The analogous results for imaginary wave number $k=i v$ are

$$
A_{j}(i v)=\chi(i v)\left(e^{j v} e^{i \delta(i v)}-e^{-j v}\right)
$$

and

$$
B_{j}(i v)=\chi_{1}(-i v)\left(e^{-j v}+e^{j v} e^{-i \delta(i v)}\right) .
$$

\section{A. One-particle form factors}

First we consider the one-particle form factors $\left\langle\Phi_{\infty}\left|\widetilde{V}_{1} X^{\dagger}(k) X^{\dagger}(i v)\right| \Phi\right\rangle$, which involve a real wave vector and the imaginary one. Since

$$
\left\langle\Phi_{\infty}\left|\widetilde{V}_{1} \mathcal{O}_{j} X^{\dagger}(i v)\right| \Phi\right\rangle=0 \quad \text { for } j=1, \ldots, M-1,
$$

inserting Eq. (3.6) gives

$$
\sum_{0<k<\pi} A_{j}(k)\left\langle\Phi_{\infty}\left|\widetilde{V}_{1} X^{\dagger}(k) X^{\dagger}(i v)\right| \Phi\right\rangle=-B_{j}(i v)\left\langle\Phi_{\infty}\left|\widetilde{V}_{1}\right| \Phi\right\rangle
$$

for $j=1, \ldots, M-1$. The sum over $k$ can be extended to $(-\pi, \pi)$ leading to the important simplification 


$$
\begin{aligned}
& \sum_{-\pi<k<\pi} \chi(k) e^{i j k}\left\langle\Phi_{\infty}\left|\widetilde{V}_{1} X^{\dagger}(k) X^{\dagger}(i v)\right| \Phi\right\rangle \\
& \quad=\left\langle\Phi_{\infty}\left|\widetilde{V}_{1}\right| \Phi\right\rangle N(i v) \chi_{1}(-i v)\left(e^{-j v}+s e^{-(M-j) v}\right)
\end{aligned}
$$

using Eqs. (3.11) and (3.12), which, in turn, suggests the definition

$$
K_{M}\left(e^{i k}\right)=\frac{\chi(k)}{N(k)} \frac{\left\langle\Phi_{\infty}\left|\tilde{V}_{1} X^{\dagger}(k) X^{\dagger}(i v)\right| \Phi\right\rangle}{\left\langle\Phi_{\infty}\left|\tilde{V}_{1}\right| \Phi\right\rangle} .
$$

Assuming that $K_{M}\left(e^{i k}\right) \rightarrow K\left(e^{i k}\right)$ suitably as $M \rightarrow \infty$ (an informal argument for the existence of such a limit is provided in Appendix A of Ref. [26]) Eqs. (3.15) and (3.16) become

$$
\frac{1}{4 \pi i} \oint_{|z|=1} d z z^{j} K(z)=N(i v) \chi_{1}(-i v) e^{-v j}
$$

for $j=1, \ldots, M-1$. Multiplying Eq. (3.17) by $t^{-j}$ with $|t|>1$, summing on $j=1$ to $M-1$, and using elementary convergence ideas reduces Eq. (3.17) to

$$
\frac{1}{4 \pi i} \oint_{|z|=1} \frac{d z}{z-t} K(z)=N(i v) \chi_{1}(-i v) \frac{1}{t e^{v}-1}
$$

This equation can be studied by Wiener-Hopf methods. Since we may assume that $K(z)$ is analytic in an annulus $r^{-1}<|z|<r$ including $|z|=1$, the Laurent theorem allows the decomposition

$$
K(z)=K_{+}(z)+K_{-}(z)
$$

with $K_{+}\left(K_{-}\right)$analytic for $|z|<r\left(|z|>r^{-1}\right)$ and from Eq. (3.17) we deduce that

$$
K_{-}(z)=\frac{2 N(i v) \chi_{1}(-i v)}{w z-1}
$$

with $w=e^{v}$. The other component $K_{+}(z)$ in Eq. (3.19) can be obtained by noting that

$$
K\left(z^{-1}\right)=-\Delta(z) K(z)
$$

with $\Delta\left(e^{i k}\right)=e^{i \delta(k)}$. Examining Eq. (2.39) shows that

$$
\Delta(z)=\frac{\Delta_{+}(z)}{\Delta_{-}(z)}
$$

where

$$
\Delta_{+}(z)=\frac{[(z-A)(z-B)]^{1 / 2}}{z-w}
$$

and

$$
\Delta_{-}(z)=\frac{[(A z-1)(B z-1)]^{1 / 2}}{w z-1} .
$$

Note that $A>1$ and that for $T<T_{c}$ we have $B>1$; thus $\Delta_{+}(z)$ is analytic for $|z|<w$ (note $w>1$ for partial wetting), whereas $\Delta_{-}(z)$ is analytic for $|z|>w^{-1}$. Equation (3.22) describes a Wiener-Hopf factorization. Using Eq. (3.22) and the Laurent decomposition (3.19) on each side of Eq. (3.21) gives

$$
\Delta_{+}(z) K_{+}(z)=-\Delta_{+}(z) K_{-}(z)-\Delta_{-}(z)\left[K_{-}\left(z^{-1}\right)+K_{+}\left(z^{-1}\right)\right] .
$$

The Hilbert transform is defined by

$$
(H f)(t)=\frac{\mathcal{P}}{\pi i} \oint_{|z|=1} \frac{d z}{z-t} f(z) .
$$

It follows directly from the development of the Laurent theorem that $(1+H) / 2$ is the projection onto the $(+)$ part in Eq. (3.19). Applying it to both sides in Eq. (3.25) gives

$$
\begin{aligned}
\Delta_{+}(z) K_{+}(z)= & -\frac{1}{2}(1+H)\left[\Delta_{+}(z) K_{-}(z)+\Delta_{-}(z) K_{-}\left(z^{-1}\right)\right] \\
& -\Delta_{-}(\infty) K_{+}(0) .
\end{aligned}
$$

Since $K_{-}(z)$ has already been evaluated as in Eq. (3.20), the Hilbert transforms in Eq. (3.27) are elementary evaluations, giving

$$
\begin{aligned}
\Delta_{+}(z) K_{+}(z)= & 2 N(i v) \chi_{1}(-i v)\left[\frac{w \Delta_{-}(w)}{z-w}+\frac{(A B)^{1 / 2}}{w}\right]-\left[\Delta_{+}(z)\right. \\
& \left.-\Delta_{+}\left(w^{-1}\right)\right] K_{-}(z)-\Delta_{+}(0) K_{+}(0) .
\end{aligned}
$$

The function $K_{+}(z)$ is given finally by putting $z=0$ in Eq. (3.28) to get $K_{+}(0)$ and then inserting this in Eq. (3.28) for $z \neq 0$. Since $K_{-}(z)$ is already known we obtain

$$
K(z)=2 \chi_{1}(-i v) N(i v) \frac{\Delta_{-}(w)}{\Delta_{+}(z)}\left[\frac{1}{w z-1}+\frac{w}{z-w}+1\right]
$$

\section{B. Two-particle form factors}

The two-particle form factors $\left\langle\Phi_{\infty}\left|\tilde{V}_{1} X^{\dagger}\left(k_{1}\right) X^{\dagger}\left(k_{2}\right)\right| \Phi\right\rangle$, which involve two real wave vectors $k_{1}$ and $k_{2}$ are investigated by considering

$$
\left\langle\Phi_{\infty}\left|\tilde{V}_{1} \mathcal{O}_{j} X^{\dagger}(k)\right| \Phi\right\rangle=0 \quad \text { for } j=1, \ldots, M-1
$$

Analogous procedures give

$$
\begin{aligned}
& \sum_{-\pi<k<\pi} N\left(k_{1}\right) \chi\left(k_{1}\right) e^{i j k_{1}}\left\langle\Phi_{\infty}\left|\tilde{V}_{1} X^{\dagger}\left(k_{1}\right) X^{\dagger}\left(k_{2}\right)\right| \Phi\right\rangle \\
& =-N(i v) \chi_{1}(-i v)\left(s e^{-(M-j) v}-e^{-j v}\right) \\
& \quad \times\left\langle\Phi_{\infty}\left|\tilde{V}_{1} X^{\dagger}\left(k_{2}\right) X^{\dagger}(i v)\right| \Phi\right\rangle \\
& \quad-N\left(k_{2}\right)\left(e^{i j k_{2}} e^{-i \delta\left(k_{2}\right)}+e^{-i j k_{2}}\right) \chi_{1}\left(k_{2}\right)\left\langle\Phi_{\infty}\left|\tilde{V}_{1}\right| \Phi\right\rangle .
\end{aligned}
$$

The natural generalization of Eq. (3.16) is 


$$
K_{M}\left(e^{i k_{1}}, e^{i k_{2}}\right)=\frac{\chi\left(k_{1}\right) \chi\left(k_{2}\right)}{N\left(k_{1}\right) N\left(k_{2}\right)} \frac{\left\langle\Phi_{\infty}\left|\tilde{V}_{1} X^{\dagger}\left(k_{1}\right) X^{\dagger}\left(k_{2}\right)\right| \Phi\right\rangle}{\left\langle\Phi_{\infty}\left|\tilde{V}_{1}\right| \Phi\right\rangle} .
$$

Multiplication of Eq. (3.30) $t^{-j},|t|>1$, with $\left|z_{2}\right|=1$ with suitable analytic assumptions on $K_{M}\left(z_{1}, z_{2}\right)$ as $M \rightarrow \infty$ (see Ref. [26]) gives

$$
\begin{aligned}
-\frac{\mathcal{P}}{4 \pi i} \int_{\left|z_{1}\right|=1} \frac{d z_{1}}{z_{1}-t} K\left(z_{1}, z_{2}\right)= & \frac{N(i v) \chi(i v)}{w t-1} K\left(z_{2}\right)+\chi\left(k_{2}\right) \chi_{1}\left(k_{2}\right) \\
& \times\left[\frac{z_{2} e^{-i \delta\left(k_{2}\right)}}{t-z_{2}}+\frac{1}{t z_{2}-1}\right] .
\end{aligned}
$$

Keeping $\left|z_{2}\right|=1$ and applying Wiener-Hopf ideas to $K\left(z_{1}, z_{2}\right)$ as a function of $z_{1}$ suggests the decomposition

$$
K\left(z_{1}, z_{2}\right)=K_{+}\left(z_{1}, z_{2}\right)+K_{-}\left(z_{1}, z_{2}\right)
$$

with $K_{+}\left(z_{1}, z_{2}\right)$ analytic inside and on $\left|z_{1}\right|=1$ and $K_{-}\left(z_{1}, z_{2}\right)$ analytic for $\left|z_{1}\right|>1$. With this in mind, Eq. (3.33) gives

$$
\begin{aligned}
K_{-}\left(z_{1}, z_{2}\right)= & 2 \chi(i v) N(i v) K\left(z_{2}\right)+4\left[\frac{e^{-i \delta\left(k_{2}\right)} z_{2}}{z_{1}-z_{2}}\right. \\
& \left.+\frac{1}{z_{1} z_{2}-1}\right] \chi\left(k_{2}\right) \chi_{1}\left(k_{2}\right)
\end{aligned}
$$

with $z_{j}=e^{i k_{j}}, j=1,2$. The analog of Eq. (3.21) is

$$
K\left(z_{1}^{-1}, z_{2}\right)=-\frac{\Delta_{+}(z)}{\Delta_{-}(z)} K\left(z_{1}, z_{2}\right)
$$

from which

$$
\begin{aligned}
\Delta_{+}\left(z_{1}\right) K_{+}\left(z_{1}, z_{2}\right)= & -\left[\Delta_{+}\left(z_{1}\right) K_{-}\left(z_{1}, z_{2}\right)+\Delta_{-}\left(z_{1}\right) K_{-}\left(z_{1}^{-1}, z_{2}\right)\right] \\
& -\Delta_{-}\left(z_{1}\right) K_{+}\left(z_{1}^{-1}, z_{2}\right)
\end{aligned}
$$

follows. Let $H_{1}$ be the Hilbert transform in the variable $z_{1}$. Then

$$
\begin{aligned}
\Delta_{+}\left(z_{1}\right) K_{+}\left(z_{1}, z_{2}\right)= & -\frac{1+H}{2}\left[\Delta_{+}\left(z_{1}\right) K_{-}\left(z_{1}, z_{2}\right)\right. \\
& \left.+\Delta_{-}\left(z_{1}\right) K_{-}\left(z_{1}^{-1}, z_{2}\right)\right]-\Delta_{-}(\infty) K_{+}\left(0, z_{2}\right)
\end{aligned}
$$

results and evaluating the Hilbert transforms using Eqs. (3.35) and (3.38) gives

$$
\begin{aligned}
\Delta_{+}\left(z_{1}\right) K_{+}\left(z_{1}, z_{2}\right)= & -2 \chi(i v) N(i v) K\left(z_{2}\right)\left[\frac{\Delta_{+}\left(z_{1}\right)-\Delta_{+}\left(w^{-1}\right)}{w z_{1}-1}\right. \\
& \left.-\frac{w \Delta_{-}(w)}{z_{1}-w}-\Delta_{-}(\infty)\right]-4 \chi\left(k_{2}\right) \chi_{1}\left(k_{2}\right) \\
& \times\left[z_{2} \Delta^{-1}\left(z_{2}\right) \frac{\Delta_{+}\left(z_{1}\right)-\Delta_{+}\left(z_{2}\right)}{z_{1}-z_{2}}\right. \\
& \left.+\frac{\Delta_{+}\left(z_{1}\right)-\Delta_{-}\left(z_{2}\right)}{z_{1} z_{2}-1}-\Delta_{-}(\infty)\left(1+\Delta\left(z_{2}\right)^{-1}\right)\right] \\
& -\Delta_{-}(\infty) K_{+}\left(0, z_{2}\right) .
\end{aligned}
$$

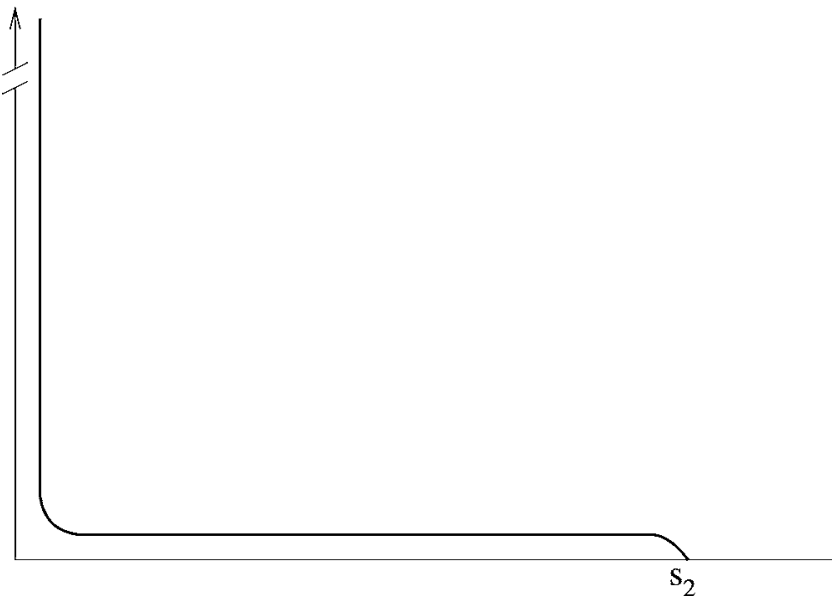

FIG. 6. Schematic depiction of the interface running from infinity along the vertical wall, bent in a corner and bound to a horizontal wall at $\left(0, s_{2}\right)$.

It is easy to check that this is indeed analytic for $\left|z_{1}\right| \leqslant 1$, as stipulated. The final step is to evaluate $K_{+}\left(0, z_{2}\right)$ and do the addition in Eq. (3.34) to get

$$
\begin{aligned}
K\left(z_{1}, z_{2}\right)= & \frac{\chi(i v)}{\chi_{1}(-i v)} K\left(z_{1}\right) K\left(z_{2}\right) \\
& +4 \chi\left(k_{2}\right) \chi_{1}\left(k_{2}\right) \frac{\Delta_{-}\left(z_{2}\right)}{\Delta_{+}\left(z_{1}\right)} \frac{z_{2}\left(z_{1}^{2}-1\right)}{\left(z_{1}-z_{2}\right)\left(z_{1} z_{2}-1\right)} .
\end{aligned}
$$

Since $K_{M}\left(z_{1}, z_{2}\right)$ is antisymmetric in its arguments, the same should be true of $K\left(z_{1}, z_{2}\right)$, but this is far from obvious in Eq. (3.40). Using results in Appendix B, it can be shown that

$$
\begin{aligned}
K\left(z_{1}, z_{2}\right)= & \frac{\chi(i v) K\left(z_{1}\right) K\left(z_{2}\right)}{\chi_{1}(-i v)} \\
& \times \frac{T\left(z_{1}, z_{2}\right)}{\left(z_{1}-z_{2}\right)\left(z_{1} z_{2}-1\right)\left(e^{-\hat{\gamma}\left(\hat{v}_{1}\right)}-w\right)\left(e^{-\hat{\gamma}\left(\hat{v}_{1}\right)}-w^{-1}\right)}
\end{aligned}
$$

with

$$
\begin{aligned}
T\left(z_{1}, z_{2}\right)= & -\left(z_{1} z_{2}+1\right)\left(z_{1}+z_{2}\right)\left(e^{-\hat{\gamma}\left(i \hat{v}_{1}\right)}-w\right)\left(e^{-\hat{\gamma}\left(i \hat{v}_{1}\right)}-w^{-1}\right) \\
& +2 e^{-\hat{\gamma}\left(i \hat{v}_{1}\right)}\left(1+z_{1}^{2}\right)\left(1+z_{2}^{2}\right)-2\left(w+w^{-1}\right)(1 \\
& \left.-e^{-2 \hat{\gamma}\left(i \hat{v}_{1}\right)}\right) z_{1} z_{2}
\end{aligned}
$$

thus confirming the antisymmetry of $K\left(z_{1}, z_{2}\right)$.

\section{RESULTS}

\section{A. Incremental free energy}

In order to evaluate the incremental free energy $F_{M}^{x}\left(s_{2}\right)$ associated with the interface which is bound to a horizontal wall at $\left(0, s_{2}\right)$ and is bent in a corner with $s_{1} \rightarrow \infty$ (see Fig. 6), we perform the spectral decompostion of $V^{\prime}$ on (2.14). When $N$ and $s_{1}$ tend to infinity only states corresponding to the largest eigenvalues contribute. Due to the linearity of the 
operators $R\left(0, s_{2}\right)$ and $R(0,0)=i \Gamma_{-1} \Gamma_{0}$ in $X^{\dagger}(k)$ and $X(k)\left(\Gamma_{-1}\right.$ is unrelated to these fermions), the vacuum term in the spectral decomposition drops out and one has to consider the one-particle states. The gap in the spectrum between the imaginary- $k$ mode with $\gamma\left(i v_{1}\right)<2\left(K_{2}-K_{1}^{*}\right)$, which is the smallest of the $\gamma^{\prime}$ s, and the real- $k$ one for which the energy is bounded below by $2\left(K_{2}-K_{1}^{*}\right)$ means that only the oneparticle term with $k=i v_{1}$ is significant. This gives for the incremental free energy

$$
\begin{aligned}
e^{-\beta F_{M}^{k}}= & e^{-\gamma\left(i v_{1}\right) s_{1}} \\
& \times \frac{\left\langle\Phi_{\infty}\left|\widetilde{V}_{1} \tilde{R}\left(0, s_{2}\right) X^{\dagger}\left(i v_{1}\right)\right| \Phi\right\rangle\left\langle\Phi\left|X\left(i v_{1}\right) R(0,0)\right| \Phi\right\rangle}{\left\langle\Phi_{\infty}\left|\widetilde{V}_{1}\right| \Phi\right\rangle} .
\end{aligned}
$$

The operator $R\left(0, s_{2}\right)$ is linear in spinors and hence in $X^{\dagger}(k)$ and $X(k)$. More precisely $R\left(0, s_{2}\right)=\Gamma_{2 s_{2}-1}$. In order to bring in the form factor $K_{M}(z)$ which we have calculated in the previous section, we need

$$
\widetilde{R}\left(0, s_{2}\right)=\tilde{V}_{1}^{-1} \Gamma_{2 s_{2}-1} \widetilde{V}_{1}=\cosh \vartheta \Gamma_{2 s_{2}-1}+i \sinh \vartheta \Gamma_{2 s_{2}} .
$$

Using again the linear expansion of $R\left(0, s_{2}\right)$ in $X^{\dagger}(k)$ and $X(k)$ (including $k=i v_{1}$ ), we obtain

$$
\begin{aligned}
e^{-\beta F_{M}^{x}\left(s_{2}\right):=} & \frac{e^{-\beta F_{M}^{x}+\gamma\left(i v_{1}\right) s_{1}}}{\mathcal{A}\left(i v_{1}\right)}=(\cosh \vartheta) \sum_{-\pi<k<\pi} N(k)^{2} e^{i s_{2} k}\left(e^{i \delta^{\prime}(k)}\right. \\
& +\tan \vartheta) \frac{K_{M}\left(e^{i k}\right)}{\chi(k)}+(\cosh \vartheta)\left(e^{i \delta^{\prime}\left(i v_{1}\right)}\right. \\
& -\tan \vartheta) e^{-s_{2} v_{1}} N\left(i v_{1}\right)+O\left(e^{-2 M v_{1}}\right)
\end{aligned}
$$

where $\quad \mathcal{A}\left(i v_{1}\right)=-N\left(i v_{1}\right) w_{1}^{-1}\left[\sinh 2 h_{1} \cosh K_{1}^{*} /\right.$ $\left.\sinh \gamma\left(i v_{1}\right)\right] e^{i \delta^{\prime}\left(i v_{1}\right)}$. The limiting result

$$
F^{x}\left(s_{2}\right)=\lim _{M \rightarrow \infty} F_{M}^{x}\left(s_{2}\right)
$$

obtained as the width of the strip diverges is

$$
\begin{aligned}
e^{-\beta F^{x}\left(s_{2}\right)}= & (\cosh \vartheta) \frac{1}{2 \pi} \int_{-\pi}^{\pi} d k e^{i s_{2} k}\left(e^{i \delta^{\prime}(k)}+\tanh \vartheta\right) \frac{K\left(e^{i k}\right)}{\chi(k)} \\
& +(\cosh \vartheta)\left(e^{i \delta^{\prime}\left(i v_{1}\right)}-\tanh \vartheta\right) e^{-s_{2} v_{1}} N\left(i v_{1}\right)
\end{aligned}
$$

In order to analyze the behavior of $F^{x}\left(s_{2}\right)$ for large $s_{2}$, the singularities of $K\left(e^{i k}\right) / \chi(k)$ are required. It is shown in Appendix B that $\chi(k)$ has simple zeros at $k= \pm i \hat{\gamma}\left(i \hat{v}_{2}\right)$ where

$$
e^{\hat{v}_{2}}=e^{2 K_{2}}\left(\cosh 2 K_{1}^{*}-\sinh 2 K_{1}^{*} \cosh 2 h_{2}\right)
$$

and $\hat{\gamma}(k)$ is the Onsager function of Eq. (1.4) with $K_{1}$ and $K_{2}$ interchanged. From Eq. (3.29), we see that $K(z)$ has "bulk" branch point singularities coming from $\Delta_{+}(z)$ through the factor $[(z-A)(z-B)]^{1 / 2}$; the branch cut at $k=i \ln B$ generates the bulk phase correlation length. The singularity at $z=w$ is removable but there is a simple pole of $K\left(e^{i k}\right)$ at $k=i v_{1}$. The features have $2 \pi$ periodicity. The "bulk" cuts in the edge wetting regime are further from the real axis than the poles just mentioned and therefore are subdominant. Using contour integral techniques we obtain

$$
\begin{aligned}
e^{-F^{x}\left(s_{2}\right)}= & I_{\text {cut }}+i(\cosh \vartheta)\left[e^{i \delta^{\prime}\left[i \hat{\gamma}\left(i \hat{v}_{2}\right)\right]}\right. \\
& +\tanh \vartheta] \frac{\chi_{1}\left(-i v_{1}\right) N\left(i v_{1}\right)}{\chi^{(\prime)}\left[i \hat{\gamma}\left(i \hat{v}_{2}\right)\right]} K\left(e^{-\hat{\gamma}\left(i \hat{v}_{2}\right)}\right) e^{-s_{2} \hat{\gamma}\left(i \hat{v}_{2}\right)} \\
& +N\left(i v_{1}\right)(\cosh \vartheta)\left[-2 e^{-v_{1}}\left(e^{i \delta^{\prime}\left(i v_{1}\right)}\right.\right. \\
& \left.+\tanh \vartheta) \frac{\chi_{1}\left(-i v_{1}\right)}{\chi\left(i v_{1}\right)}+\left(e^{i \delta^{\prime}\left(i v_{1}\right)}-\tanh \vartheta\right)\right] e^{-s_{2} v_{1}}
\end{aligned}
$$

It follows that the incremental free energy per unit length is

$$
f^{x}=\min \left(v_{1}, \hat{\gamma}\left(i \hat{v}_{2}\right)\right) .
$$

Phase transition behavior comes about because the two poles on the imaginary $k$ axis in the integrand of Eq. (4.5) cross when the temperature increases. Below the transition, the free energy behaves as $\hat{\gamma}\left(i \hat{v}_{2}\right)$, indicating that the interface is bound to both edges meeting at the corner, whereas above the transition, the free energy behaves as $v_{1}$, indicating an interface unbound from the vertical wall. The transition equation is

$$
\hat{\gamma}\left(i \hat{v}_{2}\right)=v_{1} .
$$

Taking the cosh of both sides and using the hyperbolic formula gives

$$
\cosh 2 K_{1}^{*} \cosh 2 K_{2}^{*}=\sinh 2 K_{2}^{*} \cosh \hat{v}_{2}+\sinh 2 K_{1}^{*} \cosh v_{1} .
$$

There is a special case when $h_{1}=h_{2}=h$ and $K_{1}=K_{2}=K$ which is considerably more transparent. Then Eq. (4.10) becomes

$$
e^{2 K}(\cosh 2 K-\cosh 2 h)=\sinh ^{2} 2 K .
$$

In this case an interface at the transition point crosses the lattice at the contact angle at the horizontal edge equal to $\pi / 4$; this angle decreases on increasing the temperature vanishing at wetting transition $v_{1}=0$. The phase diagram is represented by a dashed line in Fig. 4.

A further, somewhat less special case is when $K_{1}=K_{2}$, but $h_{1} \neq h_{2}$. Then Eq. (4.10) becomes

$$
2 \cosh 2 K \cosh 2 K^{*}=w_{1}+w_{1}^{-1}+w_{2}+w_{2}^{-1}
$$

with $w_{j}=e^{2 K}\left(\cosh 2 K-\cosh 2 h_{j}\right) / \sinh 2 K$. There is a special case with either $h_{1}=0$ or $h_{2}=0$, for which $w_{1}=\exp 2(K$ $-K^{*}$ ), say. Then Eq. (4.12) reads $w_{2}+1 / w_{2}=2$ so that in this case, $w_{2}=1$, which is the usual edge wetting or depinning threshold.

Some results for $h_{1} \neq h_{2}$ have been obtained numerically and are plotted in Fig. 4. It is striking that the optimal temperature suppression of the edge wetting condition with corner filling is not necessarily obtained with $h_{1}=h_{2}$, a rather counterintuitive result. 


\section{B. Location of the interface}

The location of the interface can be investigated, using the "domain wall state" idea [27] extended to the corner geometry. Such a procedure is desirable because the local magnetization cannot generally be obtained by existing means. The domain wall states $|j\rangle$, which are linear combinations of the one-particle states $|k\rangle=X_{k}^{\dagger}|\Phi\rangle$, localize the interface up to the scale of the bulk correlation length at the position $j$. The probability of a domain wall passing through $(j, n)$ is just

$$
P_{M}(j, n)=\frac{e^{-(N-n) \gamma\left(i v_{1}\right)}\left\langle\Phi_{\infty}\left|R\left(s_{2}, 0\right) \tilde{V}_{1}\left(V^{\prime}\right)^{n}\right| j\right\rangle\left\langle j\left|X^{\dagger}\left(i v_{1}\right)\right| \Phi\right\rangle}{e^{-N \gamma\left(i v_{1}\right)}\left\langle\Phi_{\infty}\left|R\left(s_{2}, 0\right) \tilde{V}_{1} X^{\dagger}\left(i v_{1}\right)\right| \Phi\right\rangle}
$$

The definition of $|j\rangle$ is

$$
|j\rangle=i \Gamma_{2 j}|\Phi\rangle .
$$

This is easy to understand if we recall the definition of the $\Gamma_{2 j}$ and the role of the Jordan-Wigner tail in reversing $x$-quantized spins. An alternative might be to look at the excess energy density, but a preliminary investigation shows that this is harder to implement than the domain wall idea. Using Eq. (4.14) and (2.41) we have

$$
\left\langle j\left|X^{\dagger}\left(i v_{1}\right)\right| \Phi\right\rangle=-i N\left(i v_{1}\right) y_{2 j}^{*}\left(i v_{1}\right) .
$$

The spectral decomposition of $V^{\prime}$ in the nominator of Eq. (4.13) with only one-particle states included gives

$$
\begin{aligned}
& \frac{\left\langle\Phi_{\infty}\left|R\left(s_{2}, 0\right) \tilde{V}_{1}\left(V^{\prime}\right)^{n}\right| j\right\rangle}{\Lambda_{0}^{n}\left\langle\Phi_{\infty}\left|\tilde{V}_{1}\right| \Phi\right\rangle} \\
& =\sum_{0<k_{2}<\pi} e^{-\gamma\left(k_{2}\right) n} \frac{\left\langle\Phi_{\infty}\left|\tilde{V}_{1} \tilde{R}\left(s_{2}, 0\right) X^{\dagger}\left(k_{2}\right)\right| \Phi\right\rangle\left\langle\Phi\left|X\left(k_{2}\right)\right| j\right\rangle}{\left\langle\Phi_{\infty}\left|\tilde{V}_{1}\right| \Phi\right\rangle} \\
& \quad+e^{-\gamma\left(i v_{1}\right) n} \frac{\left\langle\Phi_{\infty}\left|R\left(s_{2}, 0\right) \tilde{V}_{1} X^{\dagger}\left(i v_{1}\right)\right| \Phi\right\rangle}{\left\langle\Phi_{\infty}\left|\tilde{V}_{1}\right| \Phi\right\rangle}\left\langle\Phi\left|X\left(i v_{1}\right)\right| j\right\rangle .
\end{aligned}
$$

The first term on the right of Eq. (4.16) can be expressed in terms of matrix elements by using the development of $\widetilde{R}\left(s_{2}, 0\right)$ in terms of $X(k)$ and $X(k)^{\dagger}$, giving

$$
\begin{aligned}
& \frac{\left\langle\Phi_{\infty}\left|\tilde{V}_{1} \widetilde{R}\left(s_{2}, 0\right) X^{\dagger}\left(k_{2}\right)\right| \Phi\right\rangle}{(\cosh \vartheta)\left\langle\Phi_{\infty}\left|\tilde{V}_{1}\right| \Phi\right\rangle}=N\left(k_{2}\right)\left[y_{2 s_{2}-1}^{\prime *}\left(k_{2}\right)+i(\tanh \vartheta) y_{2 s_{2}}^{\prime *}\left(k_{2}\right)\right]+\sum_{-\pi<k_{1}<\pi} N\left(k_{1}\right) e^{i s_{2} k_{1}}\left(e^{i \delta^{\prime}\left(k_{1}\right)}\right. \\
& +\tanh \vartheta) \frac{\left\langle\Phi_{\infty}\left|\tilde{V}_{1} X^{\dagger}\left(k_{1}\right) X^{\dagger}\left(k_{2}\right)\right| \Phi\right\rangle}{\left\langle\Phi_{\infty}\left|\tilde{V}_{1}\right| \Phi\right\rangle}-N\left(i v_{1}\right)\left[y_{2 s_{2}-1}^{\prime}\left(i v_{1}\right)+i(\tanh \vartheta) y_{2 s_{2}}^{\prime}\left(i v_{1}\right)\right] \frac{\left\langle\Phi_{\infty}\left|\tilde{V}_{1} X^{\dagger}\left(k_{2}\right) X^{\dagger}\left(i v_{1}\right)\right| \Phi\right\rangle}{\left\langle\Phi_{\infty}\left|\tilde{V}_{1}\right| \Phi\right\rangle}
\end{aligned}
$$

Going back to Eq. (4.13) and using expressions for the eigenvectors $y^{\prime}(k)(2.34)$ and (2.35), we get

$$
\begin{aligned}
P_{M}(j, n)= & N^{2}\left(i v_{1}\right) e^{-2 j v_{1}}+N\left(i v_{1}\right) e^{\gamma\left(i v_{1}\right) n} e^{-j v_{1}} \\
& \times \frac{\left\langle\Phi_{\infty}\left|\widetilde{V}_{1}\right| \Phi\right\rangle}{\left\langle\Phi_{\infty}\left|\widetilde{V}_{1} \widetilde{R}\left(s_{2}, 0\right) X^{\dagger}\left(i v_{1}\right)\right| \Phi\right\rangle} \mathcal{G}(j, n)
\end{aligned}
$$

where

$$
\begin{aligned}
\mathcal{G}(j, n) & =\sum_{0<k_{2}<\pi} N\left(k_{2}\right) e^{-\gamma\left(k_{2}\right) n} y_{2 j}^{\prime}\left(k_{2}\right) \frac{\left\langle\Phi_{\infty}\left|\tilde{V}_{1} \tilde{R}\left(s_{2}, 0\right) X^{\dagger}\left(k_{2}\right)\right| \Phi\right\rangle}{(\cosh \vartheta)\left\langle\Phi_{\infty}\left|\tilde{V}_{1}\right| \Phi\right\rangle} \\
& =A_{M}+B_{M}+C_{M}
\end{aligned}
$$

as suggested by the three terms in Eq. (4.17). Bringing in $K_{M}\left(e^{i k_{1}}, e^{i k_{2}}\right)$ from Eq. (3.32) we get

$$
\begin{aligned}
A_{M}= & \sum_{-\pi<k_{1}, k_{2}<\pi} N^{2}\left(k_{1}\right) N^{2}\left(k_{2}\right) e^{-\gamma\left(k_{2}\right) n} e^{i j k_{2}} e^{i s_{2} k_{1}}\left(e^{i \delta^{\prime}\left(k_{1}\right)}\right. \\
& +\tanh \vartheta) \frac{K_{M}\left(e^{i k_{1}}, e^{i k_{2}}\right)}{\chi\left(k_{1}\right) \chi\left(k_{2}\right)}
\end{aligned}
$$

Taking the limit $M \rightarrow \infty$ gives

$$
A_{\infty}=\frac{1}{4 \pi^{2}} \int_{-\pi}^{\pi} d k_{1} \mathcal{P} \int_{-\pi}^{\pi} d k_{2} e^{-\gamma\left(k_{2}\right) n} e^{i j k_{2}} e^{i s_{2} k_{1}}\left(e^{i \delta^{\prime}\left(k_{1}\right)}\right.
$$

$$
+\tanh \vartheta) \frac{K\left(e^{i k_{1}}, e^{i k_{2}}\right)}{\chi\left(k_{1}\right) \chi\left(k_{2}\right)} \text {. }
$$

$B_{M}$ consists of two terms:

$$
\begin{aligned}
B_{M}= & \sum_{-\pi<k_{2}<\pi} N^{2}\left(k_{2}\right) e^{-\gamma\left(k_{2}\right) n}\left[e^{i\left(s_{2}-j\right) k_{2}}\left(e^{i \delta^{\prime}\left(k_{2}\right)}-\tanh \vartheta\right)\right. \\
& \left.+e^{-i\left(s_{2}+j\right) k_{2}} e^{i \delta\left(k_{2}\right)} e^{i k_{2}}\left(1-e^{i \delta^{\prime}\left(k_{2}\right)} \tanh \vartheta\right)\right]
\end{aligned}
$$

which in the limit $M \rightarrow \infty$ become 


$$
\begin{aligned}
B_{\infty}= & \frac{1}{2 \pi} \int_{-\pi}^{\pi} d k e^{-\gamma(k) n}\left[e^{i\left(s_{2}-j\right) k}\left(e^{i \delta^{\prime}(k)}-\tanh \vartheta\right)\right. \\
& \left.+e^{-i\left(s_{2}+j\right) k} e^{i \delta(k)} e^{i k}\left(1-e^{i \delta^{\prime}(k)} \tanh \vartheta\right)\right]
\end{aligned}
$$

Finally bringing in $K_{M}\left(e^{i k}\right)$ from Eq. (3.16) we get

$$
\begin{aligned}
C_{M}= & -e^{-s_{2} v_{1}}\left(e^{i \delta^{\prime}\left(i v_{1}\right)}\right. \\
& +\tanh \vartheta) \sum_{-\pi<k_{2}<\pi} N^{2}\left(k_{2}\right) e^{-\gamma\left(k_{2}\right) n} e^{i j k_{2}} \frac{K_{M}\left(e^{i k_{2}}\right)}{\chi\left(k_{2}\right)}
\end{aligned}
$$

and taking the limit $M \rightarrow \infty$ we find

$$
C_{\infty}=-e^{-S_{2} v_{1}}\left(e^{i \delta^{\prime}\left(i v_{1}\right)}+\tanh \vartheta\right) \int_{-\pi}^{\pi} d k e^{-\gamma(k) n} e^{i j k} \frac{K\left(e^{i k}\right)}{\chi(k)} .
$$

The expressions $A_{\infty}, B_{\infty}$, and $C_{\infty}$ are handled by noting the analytical features which the integrands contain. Both $B_{\infty}$ and $C_{\infty}$ are single integrals with simple poles coming from $K(k)$ and $e^{i \delta(k)}$, but also from the zeros of $\chi(k)$. As well we should note the existence of a steepest-descent path when both $n$ and $j$ are large, or $s_{2} \pm j$ in Eq. (4.23). In addition, the principal part integral over $k_{2}$ in the double integral of $A_{\infty}$ has simple poles at $k_{2}= \pm k_{1}$. All these facts must be taken into account, an arduous task, to extract the information given in the following section. Notice also that the quotient in front of $\mathcal{G}(j, n)$ in Eq. (4.18) is equal to $e^{\beta F^{x}\left(s_{2}\right)}$ in the limit $M \rightarrow \infty$; thus its asymptotic behavior for large $s_{2}$ is given by Eq. (4.7).

\section{Discussion of profile}

In the low temperature region, $P_{j n}$ is asymptotically constant on the straight lines

$$
n\left[\hat{v}_{2}-\gamma\left(i v_{1}\right)\right]+j\left[v_{1}-\hat{\gamma}\left(i \hat{v}_{2}\right)\right]=\mathrm{const}
$$

for large $n$ and $j$. The intercepts on the $(1,0)$ and $(0,1)$ directions both diverge as the transition is approached from the partially filled regime. This is so because the thermodynamic transition is both at the solution of Eq. (4.9) and at that of $v_{1}=i \hat{\gamma}\left(i \hat{v}_{2}\right)$. This is because

$$
\gamma\left(i v_{1}\right)=\gamma\left(i \hat{\gamma}\left(i \hat{v}_{2}\right)\right)=\hat{v}_{2}
$$

follows, a result which is not obvious a priori.

The profile dependence as exemplified by Eq. (4.26) may be understood using droplet ideas, which are based on the extension of the Andreev-Fisher droplet model [28,29] for thermodynamics via partition functions to treat correlation functions by a mesoscopic analysis of the associated droplet fluctuations. This uses Helmholtz estimates in terms of such concepts as surface tension and stiffness, as outlined in Fig. 7(a). Assuming that the boundaries of the correlation droplet of type 1 are rectilinear (on the mesoscale), a simple variational argument indicates that the angle at which the bound-

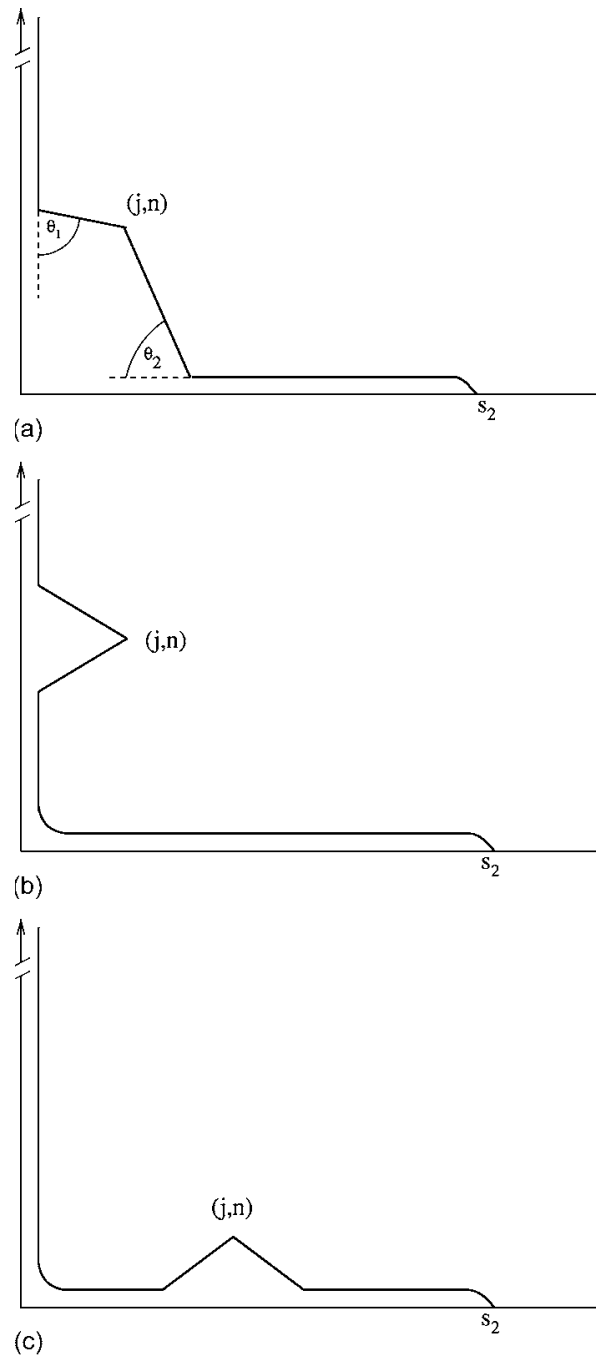

FIG. 7. Correlation droplets contributing to the interface profile (a) type 1 with droplet in the corner, $\theta_{1}$ and $\theta_{2}$ being the contact angles with walls 1 and 2, respectively, (b) type 2 isosceles triangle with base angles $\theta_{1}$, and (c) type 3 droplet, which cannot be found in the one-particle sector.

ary unbinds from each wall at the point where both intercepts diverge, is the respectivecontact angle, defined by the modified Young formula [17]. This is a simple generalization of the argument in Ref. [30].

The fluctuations of type 2 also have a droplet estimate of the same type, but with both intercepts on the $(0,1)$ axes [see Fig. 7(b)]. The shape of the correlation droplet is that of an isosceles triangle with apex at $(j, n)$ and base angles given by the contact value for $(0,1)$ partial wetting. When the implied minimization calculation is carried through, Eq. (4.26) follows with relatively little effort.

The droplet configuration of type 3 [see Fig. 7(c)], which is predicted by fluctuation analysis, cannot be obtained by the transfer matrix analysis as given because appropriate form factors for three-particle states are needed. But they could be obtained simply by rotating the transfer direction through $\pi / 2$ and repeating the previous analysis. 


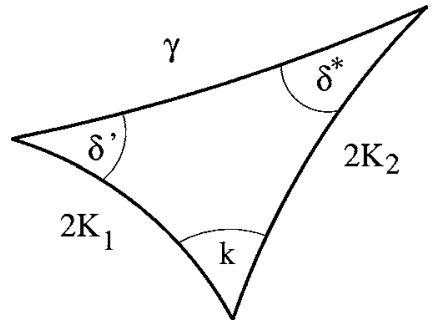

FIG. 8. Hyperbolic triangle representing the dispersion relation of the two-dimensional Ising model.

\section{CONCLUSIONS}

In this paper we have described detailed exact calculations of the filling transition in a $90^{\circ}$ corner of a quadratic Ising ferromagnetic lattice with anisotropic nearest-neighbor interactions $K_{1}$ and $K_{2}$ and different surface fields $h_{1}$ and $h_{2}$ on the abutting edges. This discussion is from both a thermodynamic and a microscopic point of view. The former confirms the conjecture on the phase diagram with $K_{1}=K_{2}$ and $h_{1}=h_{2}$ given in Ref. [20]. The latter agrees with the conjecture of Ref. [30] for the magnetization profile when partial wetting obtains.

\section{ACKNOWLEDGMENTS}

This work was partially funded by KBN Grant No. 4 T09A 06622 in Warsaw and by the EPSRC under Grant No. GR/M00426 in Oxford. D.B.A. thanks Professor K. Kaski for hospitality at LCE and HUT for partial financial support. He also thanks Professor R. Hołyst for multiple hospitality at ICHF PAN in Warsaw. The authors acknowledge valuable discussions with Professor A. O. Parry, Dr. V. Mustonen, and Dr. A. J. Wood. They also thank Dr. K. Ravilious for interesting information about Stenocara.

\section{APPENDIX A: HYPERBOLIC TRIANGLE RELATIONS}

The functions $\delta^{\prime}(k)$ and $\delta^{*}(k)$ and the wave number $k$ are the angles of the Onsager hyperbolic triangle with sides $2 K_{1}^{*}, 2 K_{2}$, and $\gamma$ shown in Fig. 8. For an Onsager triangle the hyperbolic counterpart of the al Kashi relation of a flat triangle is

$$
\cosh \gamma=\cosh 2 K_{1}^{*} \cosh 2 K_{2}-\sinh 2 K_{1}^{*} \sinh 2 K_{2} \cos k .
$$

The hyperbolic counterpart of the sine relation gives another set of useful equations:

$$
\frac{\sin k}{\sinh \gamma}=\frac{\sin \delta^{*}}{\sinh 2 K_{1}^{*}}=\frac{\sin \delta^{\prime}}{\sinh 2 K_{2}} .
$$

Applying the relations between the dual coupling constants

$$
\begin{gathered}
\sinh 2 K_{1,2}=\frac{1}{\sinh 2 K_{1,2}^{*}}, \quad \cosh 2 K_{1,2}=\frac{1}{\tanh 2 K_{1,2}^{*}} \\
\quad \cosh 2 K_{1,2}^{*}=\frac{1}{\tanh 2 K_{1,2}}
\end{gathered}
$$

to the al Kashi formula (A1) we obtain the relevant equation

$$
\cos k=\cosh 2 K_{2}^{*} \cosh 2 K_{1}-\sinh 2 K_{2}^{*} \sinh 2 K_{1} \cosh \gamma .
$$

\section{APPENDIX B: ANALYTIC STRUCTURE OF $\chi$ AND $\chi_{1}$}

From the definition (3.9), $\chi(k)$ can be written in a form suitable for factorization as

$$
\chi(k)=a_{+}\left(1+e^{i \delta^{\prime}(k)}\right)+a_{-}\left(1-e^{i \delta^{\prime}(k)}\right)
$$

with

$$
a_{ \pm}=e^{ \pm \vartheta}\left(1 \mp e^{i k}\right) / 2 \cosh \vartheta .
$$

Applying the al Kashi hyperbolic triangle formulas several times gives

$$
\begin{aligned}
2(\cosh \vartheta) \chi(k)= & \frac{\left(1+e^{i k}\right)\left(1-e^{i \delta^{\prime}}\right) e^{-\vartheta}}{(1+\cos k) \sinh 2 K_{2}}\left[\left(\sinh 2 K_{2}\right)(1+\cos k)\right. \\
& \left.+e^{2 \vartheta}(\sinh \gamma)\left(1+\cos \delta^{\prime}\right)\right]
\end{aligned}
$$

Expressing $\cos k$ in terms of $\cosh \gamma$ by Eq. (A1) brings Eq. (B3) to the quadratic in $e^{\gamma}$ :

$$
2(\cosh \vartheta) \chi(k)=2 \frac{\left(1-e^{i \delta^{\prime}}\right) e^{-\gamma}\left(e^{\gamma}-\hat{w}_{2}\right)\left(e^{\gamma}-\hat{A}^{-1}\right) e^{-\vartheta}}{\left(1+e^{-i k}\right) \sinh 2 K_{1}^{*} \sinh 2 K_{2} \sinh ^{2} h_{2}}
$$

where

$$
\hat{w}_{2}=e^{2 K_{2}}\left(\cosh 2 K_{1}^{*}-\sinh 2 K_{1}^{*} \cosh 2 h_{2}\right) .
$$

The analogous result for $\chi_{1}(k)$ is

$2\left(\cosh \vartheta \chi_{1}\right)(k)$

$$
=i \frac{\left(1+e^{i k}\right)\left(1-e^{i \delta^{\prime}}\right)\left(\hat{w}_{2} e^{\gamma}-1\right)\left(e^{\gamma}-\hat{B}\right) e^{-\gamma} e^{-\vartheta} e^{-2 K_{2}}}{2 \sin k \sinh 2 K_{1}^{*} \sinh 2 K_{2} \sinh ^{2} h_{2}} .
$$

From Eqs. (B4) and (B5), the singularities at the zeros of $\left(1+e^{-i k}\right)$ are removable. There are bulk branch points and simple zeros at $e^{\gamma(k)}=\hat{w}_{2}$ and $e^{\gamma(k)}=\hat{A}^{-1}$. With the notation

$$
\hat{w}_{2}=e^{\hat{v}_{2}}
$$

and use of Appendix A, the first zeros are simple and at

$$
k= \pm i \hat{\gamma}\left(i \hat{v}_{2}\right)
$$

with $2 \pi$-periodic repetition. The second set of zeros from $\gamma(k)=-2\left(K_{1}^{*}+K_{2}\right)$ are on the second sheet in the $e^{i k}$ plane. The following two formulas will be useful:

$$
\begin{aligned}
& \frac{\chi(k)}{\chi\left(i v_{1}\right)} \\
& \quad=\frac{\left(1+e^{v_{1}}\right)\left(1-e^{i \delta^{\prime}}\right) e^{-\gamma}\left(e^{\gamma}-\hat{w}_{2}\right)\left(e^{\gamma}-\hat{A}^{-1}\right)}{\left(1+e^{-i k}\right)\left(1-e^{i \delta^{\prime}\left(i v_{1}\right)}\right) e^{-\gamma\left(i v_{1}\right)}\left(e^{\gamma\left(i v_{1}\right)}-\hat{w}_{2}\right)\left(e^{\gamma\left(i v_{1}\right)}-\hat{A}^{-1}\right)},
\end{aligned}
$$




$$
\frac{\chi_{1}(k)}{\chi_{1}\left(i v_{1}\right)}=\frac{\left(1+e^{-i k}\right)\left(1-e^{i \delta^{\prime}}\right) e^{-\gamma}\left(\hat{w}_{2} e^{\gamma}-1\right)\left(e^{\gamma}-\hat{B}\right)}{\left(1+e^{i v_{1}}\right)\left(1-e^{i \delta^{\prime}\left(i v_{1}\right)}\right) e^{-\gamma\left(i v_{1}\right)}(\sin k)\left(\hat{w}_{2} e^{\gamma(i v)}-1\right)\left(e^{\gamma\left(i v_{1}\right)}-\hat{B}\right)} .
$$

These give the result

$$
\frac{\chi(k) \chi_{1}(k)}{\chi\left(i v_{1}\right) \chi_{1}\left(i v_{1}\right)}=\frac{\left[1-\left(\cos \delta^{\prime}(k)\right)\right]\left(\sin i v_{1}\right) e^{-2 \gamma(k)}\left(e^{\gamma(k)}-\hat{w}_{2}\right)\left(\hat{w}_{2} e^{\gamma(k)}-1\right)}{\left[1-\left(\cos \delta^{\prime}\left(i v_{1}\right)\right)\right] \sin k e^{-2 \gamma\left(i v_{1}\right)}\left(e^{\gamma\left(i v_{1}\right)}-\hat{w}_{2}\right)\left(\hat{w}_{2} e^{\gamma\left(i v_{1}\right)}-1\right)} \times \frac{\left(e^{\gamma(k)}-\hat{A}^{-1}\right)\left(e^{\gamma(k)}-\hat{B}\right)}{\left(e^{\gamma\left(i v_{1}\right)}-\hat{A}^{-1}\right)\left(e^{\gamma\left(i v_{1}\right)}-\hat{B}\right)},
$$

which may be simplified further by considering

$$
\frac{\left(1-\cos \delta^{\prime}\right) e^{-\gamma}}{\sin k}\left(e^{\gamma}-\hat{A}^{-1}\right)\left(e^{\gamma}-\hat{B}\right)=\frac{(\sinh \gamma)\left(1-\cos \delta^{\prime}\right) e^{-\gamma}\left(e^{\gamma}-\hat{A}^{-1}\right)\left(e^{\gamma}-\hat{B}\right)}{\sinh ^{2} k} \frac{\sin k}{\sinh \gamma} .
$$

Using the al Kashi formulas and factorizing $\sin ^{2} k$ in $e^{\gamma}$ dramatically simplifies Eqs. (B11) and (B12) giving

$$
\frac{\chi(k) \chi_{1}(k)}{\chi\left(i v_{1}\right) \chi_{1}\left(i v_{1}\right)}=\frac{\sin k}{\sinh \gamma} \frac{\left(\sinh \gamma\left(i v_{1}\right)\right)}{\sin i v_{1}} \frac{\cosh \gamma-\cosh \hat{v}_{2}}{(\cosh \gamma)\left(i v_{1}\right)-\cosh \hat{v}_{2}}
$$

needed in the analysis of Eq. (4.21).

[1] For a general review of wetting see, for example, D. E. Sullivan and M. M. Telo da Gama, in Fluid Interfacial Phenomena, edited by C. A. Croxton (Wiley, New York, 1985); S. Dietrich, in Phase Transitions and Critical Phenomena, edited by C. Domb and J. L. Lebowitz (Academic Press, London, 1988), Vol. 12, p. 1.

[2] See, for example, S. Dietrich, in New Approaches to Old and New Problems in Liquid State Theory-Inhomogeneities and Phase Separation in Simple, Complex and Quantum Fluids, edited by C. Caccamo, J. P. Hansen, and G. Stell, NATO Advanced Studies Institute, Ser. B: Physics (Kluwer, Dordrecht, 1998), pp. 197-244, and references therein.

[3] P. Concus and R. Finn, Proc. Natl. Acad. Sci. U.S.A. 63, 292 (1969).

[4] Y. Pomeau, J. Colloid Interface Sci. 113, 5 (1986).

[5] E. H. Hauge, Phys. Rev. A 46, 4994 (1992).

[6] K. Rejmer, S. Dietrich, and M. Napiórkowski, Phys. Rev. E 60, 4027 (1999).

[7] A. R. Parker and C. R. Lawrence, Nature (London) 414, 33 (2001).

[8] M. E. Fisher, J. Phys. Soc. Jpn. 26 (Suppl.), 87 (1969).

[9] L. Onsager, Phys. Rev. 65, 117 (1944).

[10] D. B. Abraham and N. M. Svrakić, Phys. Rev. Lett. 56, 1172 (1986).

[11] R. L. Dobrushin, Theor. Probab. Appl. 17, 582 (1972).

[12] D. B. Abraham and P. Reed, Commun. Math. Phys. 49, 35 (1976).

[13] M. P. A. Fisher, D. S. Fisher, and J. D. Weeks, Phys. Rev. Lett. 48, 368 (1982)
[14] D. G. A. L. Aarts, M. Schmidt, and H. N. W. Lekkerkerker, Science 304, 847 (2004).

[15] D. B. Abraham and M. E. Issigoni, J. Phys. A 13, L89 (1980).

[16] M. E. Fisher, J. Stat. Phys. 34, 667 (1984).

[17] D. B. Abraham and L. F. Ko, Phys. Rev. Lett. 63, 275 (1989).

[18] D. B. Abraham and P. J. Upton, Phys. Rev. B 37, R3835 (1988).

[19] G. Wulff, Z. Kristallogr. 34, 449 (1901).

[20] A. O. Parry, A. J. Wood, E. Carlon, and A. Drzewiński, Phys. Rev. Lett. 87, 196103 (2001).

[21] D. B. Abraham and A. Maciołek, Phys. Rev. Lett. 89, 286101 (2002).

[22] A. O. Parry, C. Rascón, and A. J. Wood, Phys. Rev. Lett. 83, 5535 (1999).

[23] B. Kaufman, Phys. Rev. 76, 1232 (1949).

[24] A. Maciołek, J. Phys. A 29, 3837 (1996).

[25] P. Jordan and E. P. Wigner, Z. Phys. 47, 631 (1928).

[26] D. B. Abraham and F. T. Latrémoliére, J. Stat. Phys. 81, 539 (1995).

[27] D. B. Abraham and F. T. Latrémoliére, Phys. Rev. Lett. 77, 171 (1996)

[28] A. F. Andreev, Zh. Eksp. Teor. Fiz. 45, 2068 (1963) [Sov. Phys. JETP 18, 1415 (1964)].

[29] M. E. Fisher, Physics (Long Island City, N.Y.) 3, 255 (1967); in Critical Phenomena, Proceedings of the International School of Physics "Enrico Fermi," Course LI, edited by M. S. Green (Academic, New York, 1971), p. 1.

[30] D. B. Abraham, A. O. Parry, and A. J. Wood, Europhys. Lett. 60, 106 (2002). 